\title{
Branching random walk with trapping zones
}

\author{
Romain Biard $^{\mathrm{a}}$, Bastien Mallein ${ }^{\mathrm{b}}$, Landy Rabehasaina ${ }^{\mathrm{a}}$ \\ ${ }^{a}$ LMB, UFR-ST, 16 route de Gray, 25030 Besançon cédex, France. Email: \\ romain.biard@univ-fcomte.fr, lrabehas@univ-fcomte.fr, Laboratoire de Mathématiques, \\ Université Bourgogne Franche-Comté. \\ ${ }^{b}$ Winterthurerstrasse 190 CH-8057 Zürich, Switzerland. Email: \\ bastien.mallein@math.uzh.ch, Institut für Mathematik, Universität Zürich.
}

\begin{abstract}
We consider a branching random walk with values in a certain set $\mathbb{S}$, where the branching mechanism is different according to whether particles (individuals) are in a certain so called trapping set $A \subset \mathbb{S}$ or not. We are then interested, under different scenarios, in properties of either the transient random measure describing distribution of individuals on $\mathbb{S}$ over time or its asymptotic behaviour.
\end{abstract}

Keywords: Branching random walk, absorbing set, Galton Watson process. MSC classification: 60J25, 60G22, 60K30.

\section{Introduction}

This paper was originally motivated by the modelling and studying of evolution of Transposable Elements within a DNA strand. Transposable elements are small DNA sequences (with negligible size) that have the ability to change their position through a so called cut-and-paste procedure, and also to create copies (copy-and-paste) within the genome, throughout successive generations. One particular feature of the DNA strand is that when a transposable element lives in the centromere, it loses its cut-and-paste and copy-and-paste abilities, as the centromere is an inactive area. In this paper, we have chosen to model the evolution of those transposable elements by a particular random tree and branching random walk. Individuals of this random tree live in a subset $\mathbb{S}$ of the real line. The inactive centromere area is modelled by a subset of $\mathbb{S}$ within which spawned transposable elements remain there forever. 
Mathematically, let $\mathbf{T}$ be a random tree. We consider in this paper a branching random walk $(V(x), x \in \mathbf{T})$ on a set $\mathbb{S} \subset \mathbb{R}$ starting from $a \in \mathbb{S}$. Following standard notation (see e.g. $[1,9]$ ), we let $V(x) \in \mathbb{S}$ the position of particle $x,|x|$ its generation, and $x_{k} \in \mathbf{T}$ the ancestor of $x$ at generation $k \leq|x|$. For any $a \in \mathbb{S}$, we write $\mathbb{P}_{a}$ the law of $(V(x), x \in \mathbf{T})$ with associated expectation $\mathbb{E}_{a}$, or $\mathbb{P}$ and $\mathbb{E}$ when no confusion is possible.

The originality of our model is the presence of a trapping zone $A \subset \mathbb{S}$. When an individual first enters this set, it is immediately frozen and does not reproduce any more. Such an individual will be called inactive, as opposed to an offspring in $\bar{A}$ which will be called active. For each generation, an active offspring disappears with probability $q \in(0,1)$ or survives with probability $p=1-q$. Whenever it survives, it stays at the same position and can create randomly $N \in \mathbb{N}$ children and the displacement from the originally offspring to a child follows a random variable with distribution $V$, see Figure 1 . We

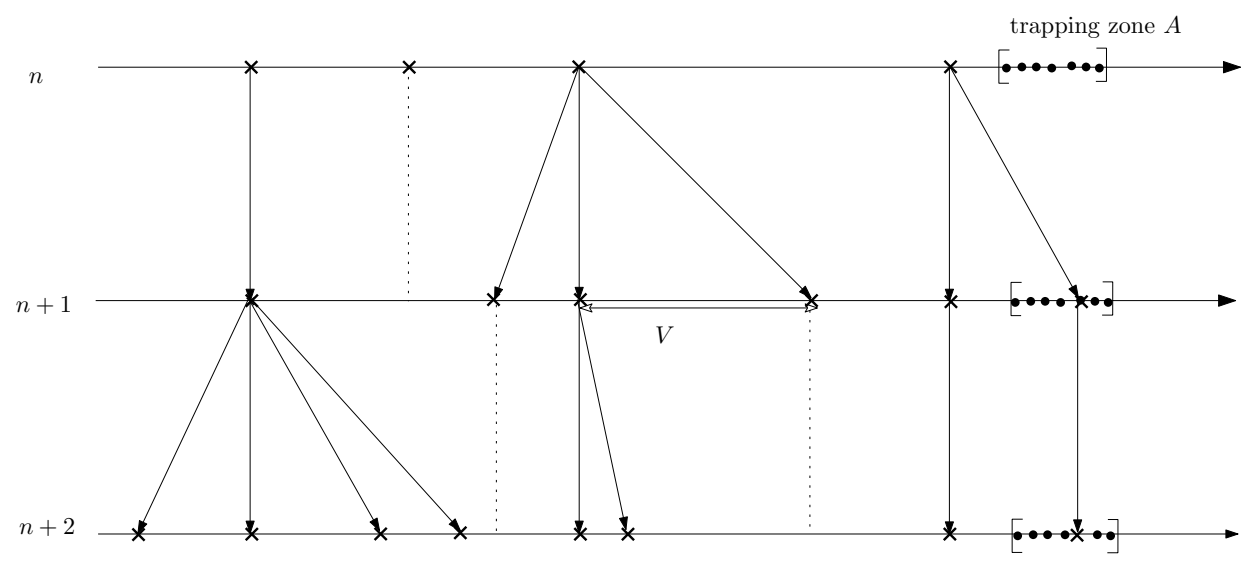

Figure 1: Evolution of branching random walk with trapping zones.

also note that this process behaves like two traditional branching random walks with distinct point process distributions with respective displacements distributed as 0 or $V$ according to whether particles are in $A$ or $\bar{A}$. We will make the following assumptions:

- generation of offsprings are independent from displacements,

- distribution $V$ of displacements is symmetric, i.e. $-V \stackrel{\mathcal{D}}{=} V$, and is light tailed, i.e. admits exponential moments $\mathbb{E}\left(e^{u V}\right)$ for $u$ in some interval $I \subset \mathbb{R}$ that contains zero, 
- branching random walk is supercritical while in $\bar{A}$, i.e.

$$
m:=p(1+\mathbb{E} N)>1 .
$$

We introduce the following random measures $\nu_{n}, \nu_{n}^{(1)}, \nu_{n}^{(2)}$ defined for any non negative measurable function $f: \mathbb{S} \longrightarrow[0,+\infty)$ and $x \in \mathbf{T}$, as well as exit time $T_{A}(x)$ as:

$$
\begin{aligned}
\nu_{n}(f) & =\sum_{|x|=n} f(V(x)), \\
T_{A}(x) & =\min \left\{k \leq|x|: V\left(x_{k}\right) \in A\right\}, \\
\nu_{n}^{(1)}(f) & =\sum_{|x|=n} f(V(x)) \mathbb{1}_{\left\{T_{A}(x)>n\right\}}, \\
\nu_{n}^{(2)}(f) & =\sum_{|x|=n} f(V(x)) \mathbb{1}_{\left\{T_{A}(x) \leq n\right\}}=\sum_{|x| \leq n} f(V(x)) \mathbb{1}_{\left\{T_{A}(x)=|x|\right\}} .
\end{aligned}
$$

$T_{A}(x)$ is the time at which individual the first ancestor of $x$ entered the trapping zone $A$, which is equal to $+\infty$ if the individual is still active. Random measure $\nu_{n}=\sum_{|x|=n} \delta_{V(x)}$ represents the distribution of the population alive (both active and inactive) at time $n$. Similarly, $\nu_{n}^{(1)}$ and $\nu_{n}^{(2)}$ represent respectively distribution of active and inactive individuals in the process, so that one in particular has

$$
\nu_{n}(f)=\nu_{n}^{(1)}(f)+\nu_{n}^{(2)}(f) .
$$

Letting $f \equiv 1$ in $(2)$, we in particular note that

$$
\begin{aligned}
\nu_{n}(1) & =\sum_{|x|=n} 1=\#\{x,|x|=n\}, \\
\nu_{n}^{(1)}(1) & =\sum_{|x|=n} \mathbb{1}_{\left\{T_{A}(x)>n\right\}}=\#\{x,|x|=n, x \in \bar{A}\}, \\
\nu_{n}^{(2)}(1) & =\sum_{|x|=n} \mathbb{1}_{\left\{T_{A}(x) \leq n\right\}}=\#\{x,|x|=n, x \in A\}
\end{aligned}
$$

are respectively the number of offspring alive, active and inactive at the $n$th generation. Finally, we define the so-called Laplace exponent of the branching random walk in $\bar{A}$ as

$$
m(u):=p\left[1+\mathbb{E}(N)\left(\mathbb{E} e^{u V}\right)\right]
$$

for $u \in I$. Note in particular that $m(u)=\mathbb{E}\left[\sum_{|x|=1} e^{u \tilde{V}(x)}\right]$ where $(\tilde{V}(x), x \in$ $\mathbf{T}$ ) is a branching random walk where particles evolve in $\mathbb{S}$ (without trapping 
zone), and with displacement distributed as $V$. Also note that $m$ defined in (1) verifies $m=m(0)$.

Notation. In the following, convergence of a random measure $\mu_{n}$ towards $\mu_{\infty}$ on a set $E$ such that $\mathbb{P}(E)>0$ will be denoted by

$$
\mu_{n} \underset{n \rightarrow+\infty}{\rightsquigarrow} \mu_{\infty} \text { on } E,
$$

meaning that for any continuous non-negative function $f: \mathbb{S} \longrightarrow[0,+\infty)$, $\int_{\mathbb{S}} f(x) \mu_{n}(d x) \longrightarrow \int_{\mathbb{S}} f(x) \mu_{\infty}(d x)$ as $n \rightarrow \infty$ on $E$.

\section{Organization of the paper and main results}

In this paper, quantities of interest are mainly quantities related to the measure $\nu_{n}$.

- Let

$$
\psi_{n}=\psi_{n}(u, a):=\mathbb{E}_{a} \nu_{n}\left(e^{u \cdot}\right) .
$$

In Section 3, we assume the convergence of $\tilde{\psi}_{n}(A) / \kappa^{n}$ for some measure $\tilde{\psi}_{n}(d y)$ defined on $\mathbb{\$}$ related to the branching random walk, and for a convenient choice of $\kappa$. We show that this convergence implies convergence of $\psi_{n} / \kappa^{n}$ for all $u$ lying in interval $I$ and obtain its expression under mild assumptions. Also, the generating function of $\left(\psi_{n}\right)_{n \in \mathbb{N}}$,

$$
\hat{\psi}(t)=\hat{\psi}(t, u, a):=\sum_{n=0}^{\infty} t^{n} \mathbb{E}_{a}\left(\nu_{n}\left(e^{u \cdot}\right)\right)=\sum_{n=0}^{\infty} t^{n} \psi_{n}
$$

for $|t|$ small enough such that the series converges, is studied.

- In Section 4 , in the case $\mathbb{S}=\mathbb{Z}$ and $\bar{A}$ a finite set, we show that, on the set of survival of the branching process,

$$
\frac{\nu_{n}}{\nu_{n}(1)} \underset{n \rightarrow+\infty}{\rightsquigarrow} \mu_{\infty}
$$

for some deterministic probability measure $\mu_{\infty}$.

- In Section 5 , in the case $\mathbb{S}=\mathbb{Z}, \bar{A}$ a finite set, and $V$ Rademacher distributed, computations for (3), (4) and (5) are detailed and some explicit results are given. We also get the asymptotic behaviour of the expected number of particles in the trap zone $A$ and its complementary set. 
- In Section 6 , in the case $\mathbb{S}=\mathbb{R}, \bar{A}=(-1,1)$ and $V$ Laplace distributed, the generating function (4) is obtained in a closed form.

- Finally, in Section 7, the case where $\mathbb{S}$ is a torus is investigated, and a functional equation for $a \mapsto \hat{\psi}(t, u, a)$ in (4) is exhibited when $V$ is Laplace distributed.

Remark 1. Note that convergence of $\psi_{n} / \kappa^{n}$ for all $u \in I$ is equivalent to convergence as $n \rightarrow \infty$ of measure $\mathbb{E} \frac{\nu_{n}}{\kappa^{n}}$ defined by $\mathbb{E} \frac{\nu_{n}}{\kappa^{n}}(f)=\mathbb{E}\left(\int_{\mathbb{S}} f(x) \frac{\nu_{n}(d x)}{\kappa^{n}}\right)$ for all measurable function $f: \mathbb{S} \longrightarrow[0,+\infty)$.

We finish this section by recalling the following result, which gives a link between the branching random walk $(\tilde{V}(x), x \in \mathbf{T})$ on $\mathbb{S}$ with associated displacement $V$ and a certain random walk $\left\{S_{n}, n \in \mathbb{N}\right\}$, whose history can be tracked back at least to [6].

Lemma 2 (Many to one Lemma). Let $\left\{S_{n}, n \in \mathbb{N}\right\}$ be the one dimensional random walk defined by

$$
S_{n}:=a+\sum_{k=1}^{n} \xi_{k}, \quad n \geq 1
$$

where $\left(\xi_{k}\right)_{k \in \mathbb{N}}$ is i.i.d. with distribution characterized by

$$
\mathbb{E}\left(f\left(\xi_{k}\right)\right)=\frac{1}{m} \mathbb{E}\left(\sum_{|x|=1} f(\tilde{V}(x))\right)=\frac{1}{m} p[f(0)+\mathbb{E}(N) \mathbb{E}(f(V))]
$$

for all measurable non negative function $f: \mathbb{S} \longrightarrow[0,+\infty)$. Then for all measurable non negative function $\varphi: \mathbb{S}^{n} \longrightarrow \mathbb{R}$, one has

$$
\mathbb{E}_{a}\left(\sum_{|x|=n} \varphi\left(\tilde{V}\left(x_{1}\right), \ldots, \tilde{V}\left(x_{n}\right)\right)\right)=m^{n} \mathbb{E}\left(\varphi\left(S_{1}, \ldots, S_{n}\right)\right)
$$

where we recall that $a$ is the starting location of the branching random walk.

With a slight abuse of notation, we will, similarly to (2), denote for any set $C \subset \mathbb{S}$

$$
T_{C}:=\inf \left\{n \geq 0, S_{n} \notin C\right\}
$$

the exit time out of $C$ of random walk $\left\{S_{n}, n \in \mathbb{N}\right\}$. 


\section{General results}

Remembering that an element in $A$ will stay there forever without having further offspring, and decomposing $\psi_{n}$ according to whether an element $x$ is in $A$, if so, when its first ancestor was in $A$ for the first time, we have :

$$
\begin{aligned}
\psi_{n} & =\mathbb{E}\left[\sum_{|x|=n} e^{u V(x)} \mathbb{1}_{\left\{T_{A}(x)>n\right\}}\right]+\mathbb{E}\left[\sum_{|x|=n} e^{u V(x)} \mathbb{1}_{\left\{T_{A}(x) \leq n\right\}}\right], \\
& =\mathbb{E} \nu_{n}^{(1)}\left(e^{u \cdot}\right)+\mathbb{E} \nu_{n}^{(2)}\left(e^{u \cdot}\right) \\
& :=\psi_{n}^{(1)}+\psi_{n}^{(2)}
\end{aligned}
$$

We now introduce the following measure on $\mathbb{S}$

$$
\tilde{\psi}_{n}(d y)=\mathbb{E}\left[\sum_{|z|=n} e^{u V(z)} \mathbb{1}_{\left\{T_{A}(z)>n-1\right\} \cap\{V(z) \in d y\}}\right], n \geq 1,
$$

with $\tilde{\psi}_{0}(d y)=e^{u a} \delta_{a}(d y)$, so that we have

$$
\psi_{n}^{(1)}=\tilde{\psi}_{n}(\bar{A})
$$

and

$$
\psi_{n}^{(2)}=\sum_{k=0}^{n} \tilde{\psi}_{k}(A) .
$$

Let us assume there exists $\kappa>0$ such that the following limit exists:

$$
\tilde{\psi}^{(\kappa)}(A):=\lim _{n \rightarrow+\infty} \frac{1}{\kappa^{n}} \tilde{\psi}_{n}(A) .
$$

We then have

$$
\frac{1}{\kappa^{n}} \psi_{n}^{(2)}=\frac{1}{\kappa^{n}} \sum_{k=0}^{n} \tilde{\psi}_{k}(A)=\sum_{k=0}^{\infty} \mathbb{1}_{k \leq n} \frac{1}{\kappa^{k}} \frac{1}{\kappa^{n-k}} \tilde{\psi}_{n-k}(A) .
$$

Remark 3. Existence of a $\kappa$ such that (12) converges is not a trivial topic in general. Nevertheless, an example is given in Section 5, where we explicit a $\kappa$ such that (12) converges for the particular case of Rademacher distribution for the displacements. 
Proposition 4. Under Condition (12) and assumption $\kappa>1$, we have

$$
\tilde{\psi}^{(\kappa)}(\bar{A})=\frac{\kappa}{m(u)-\kappa} \tilde{\psi}^{(\kappa)}(A)
$$

and, if $\kappa$ in addition satisfies $\kappa<m$,

$$
\psi^{(\kappa)}:=\lim _{n \rightarrow \infty} \frac{1}{\kappa^{n}} \psi_{n}=\frac{\kappa}{\kappa-1} \frac{m(u)-1}{m(u)-\kappa} \tilde{\psi}^{(\kappa)}(A) .
$$

Proof. Using Relation (9) as well as the dominated convergence theorem in (13), we obtain the following limit as $n$ tends to infinity, provided that $\frac{1}{\kappa^{n}} \tilde{\psi}_{n}(\bar{A})$ converges to some limit $\tilde{\psi}^{(\kappa)}(\bar{A})$ as $n \rightarrow \infty$ under a suitable assumption on $\kappa$ which will be made precise below (namely, that $\kappa<m$ ):

$$
\begin{aligned}
\psi^{(\kappa)}:=\lim _{n \rightarrow+\infty} \frac{1}{\kappa^{n}} \psi_{n} & =\tilde{\psi}^{(\kappa)}(\bar{A})+\sum_{k=0}^{\infty} \frac{1}{\kappa^{k}} \tilde{\psi}^{(\kappa)}(A), \\
& =\tilde{\psi}^{(\kappa)}(\bar{A})+\frac{\kappa}{\kappa-1} \tilde{\psi}^{(\kappa)}(A) .
\end{aligned}
$$

The above equality yields expression of limit $\psi^{(\kappa)}$ if both quantities $\tilde{\psi}^{(\kappa)}(\bar{A})$ and $\tilde{\psi}^{(\kappa)}(\bar{A})$ are known. So, let us prove that $\tilde{\psi}^{(\kappa)}(\bar{A})$ indeed exists and establish a relation between these two latter quantities. We note that we have $\tilde{\psi}_{n}(A)+\tilde{\psi}_{n}(\bar{A})=\mathbb{E}\left[\sum_{|z|=n} e^{u V(z)} \mathbb{1}_{\left\{T_{A}(z)>n-1\right\}}\right]$. Remembering that in $\bar{A}$ the model evolves like a normal branching random walk, we may then use Lemma 2 and write

$$
\begin{aligned}
\mathbb{E}\left[\sum_{|z|=n} e^{u V(z)} \mathbb{1}_{\left\{T_{A}(z)>n-1\right\}}\right] & =m^{n} \mathbb{E}\left[e^{u S_{n}} \mathbb{1}_{\left\{S_{j} \in \bar{A}, j \leq n-1\right\}}\right] \\
& =m^{n} \mathbb{E}\left[e^{u \xi_{n}}\right] \mathbb{E}\left[e^{u S_{n-1}} \mathbb{1}_{\left\{S_{j} \in \bar{A}, j \leq n-1\right\}}\right] \\
& =m \mathbb{E}\left[e^{u \xi_{n}}\right] \mathbb{E}\left[\sum_{|z|=n-1} e^{u V(z)} \mathbb{1}_{\left\{T_{A}(z)>n-1\right\}}\right] \\
& =m \mathbb{E}\left[e^{u \xi_{n}}\right] \tilde{\psi}_{n-1}(\bar{A}) .
\end{aligned}
$$

Let us remember that one has by Definition (7) that $\mathbb{E}\left[e^{u \xi_{n}}\right]=\frac{m(u)}{m}$, so that one obtains from (17) that

$$
\tilde{\psi}_{n}(A)+\tilde{\psi}_{n}(\bar{A})=m(u) \tilde{\psi}_{n-1}(\bar{A}) .
$$


Let us then prove that $\frac{1}{\kappa^{n}} \tilde{\psi}_{n}(\bar{A})$ converges to some limit $\tilde{\psi}^{(\kappa)}(\bar{A})$ as $n \rightarrow \infty$ if we suppose that $\kappa<m(u)$. Dividing (18) by $m(u) \kappa^{n-1}$ yields that

$$
\begin{aligned}
\frac{\tilde{\psi}_{n-1}(\bar{A})}{\kappa^{n-1}} & =\frac{\kappa}{m(u)} \frac{\tilde{\psi}_{n}(\bar{A})}{\kappa^{n}}+\frac{\kappa}{m(u)} \frac{\tilde{\psi}_{n}(A)}{\kappa^{n}} \\
& =\sum_{j=1}^{\infty}\left(\frac{\kappa}{m(u)}\right)^{j} \frac{\tilde{\psi}_{n-1+j}(A)}{\kappa^{n-1+j}}
\end{aligned}
$$

the above series converging because $\kappa / m(u)<1$ and $\frac{\tilde{\psi}_{n}(A)}{\kappa^{n}}$ converges as $n \rightarrow \infty$. Letting $n \rightarrow \infty$ in (19) and using the dominated convergence theorem yields that $\tilde{\psi}^{(\kappa)}(\bar{A})=\lim _{n \rightarrow \infty} \frac{1}{\kappa^{n}} \tilde{\psi}_{n}(\bar{A})$ exists and that (14) holds. Note that condition $\kappa<m(u)$ for all $u \in I$ is in fact equivalent to $\kappa<m$. (16) and (14) thus imply (15) after some simplification.

To finish this subsection, the following proposition gives the expression of the generating function of $\left(\psi_{n}\right)_{n \in \mathbb{N}}$ in function of the generating function of $\left(\tilde{\psi}_{n}(A)\right)_{n \in \mathbb{N}}$.

Proposition 5. Let $\hat{\psi}(t, A)=\hat{\psi}(t, u, a, A):=\sum_{n=0}^{\infty} t^{n} \tilde{\psi}_{n}(A)$ the generating function of $\left(\tilde{\psi}_{n}(A)\right)_{n \in \mathbb{N}}$, which we assume is defined for $|t|$ small enough. Generating function defined in (4) has the following expression

$$
\hat{\psi}(t)=\frac{\hat{\psi}(t, A)-e^{u a}}{m(u) t-1}+\frac{1}{1-t} \hat{\psi}(t, A), \quad t<\frac{1}{m(u)} .
$$

Proof. Let $\hat{\psi}(t, \bar{A})$ be likewise defined as $\hat{\psi}(t, A)$. The following is obtained thanks to Relation (18), that yields the following relation between these two quantities

$$
\hat{\psi}(t, A)+\hat{\psi}(t, \bar{A})=\tilde{\psi}_{0}(A)+\tilde{\psi}_{0}(\bar{A})+m(u) t \hat{\psi}(t, \bar{A})
$$

with $\tilde{\psi}_{0}(A)+\tilde{\psi}_{0}(\bar{A})=e^{u a}$, so that one has the following expression

$$
\hat{\psi}(t, \bar{A})=\frac{\hat{\psi}(t, A)-e^{u a}}{m(u) t-1}
$$

where $|t|<1 / m(u)$. Now, similarly to (9), (10) and (11), decomposing to when an element $x$ in $A$ had its first ancestor in that set for the first time, we have

$$
\mathbb{E}\left[\nu_{n}\left(e^{u \cdot}\right)\right]=\tilde{\psi}_{n}(\bar{A})+\sum_{k=0}^{n} \tilde{\psi}_{k}(A)=\tilde{\psi}_{n}(\bar{A})+\sum_{k=0}^{n} \tilde{\psi}_{n-k}(A)
$$


whence arrive at

$$
\begin{aligned}
\hat{\psi}(t) & =\sum_{n=0}^{\infty} t^{n} \tilde{\psi}_{n}(\bar{A})+\sum_{n=0}^{\infty} t^{n}\left[\sum_{k=0}^{n} \tilde{\psi}_{n-k}(A)\right] \\
& =\sum_{n=0}^{\infty} t^{n} \tilde{\psi}_{n}(\bar{A})+\sum_{n=0}^{\infty}\left[\sum_{k=0}^{n} t^{k} t^{n-k} \tilde{\psi}_{n-k}(A)\right] \\
& =\sum_{n=0}^{\infty} t^{n} \tilde{\psi}_{n}(\bar{A})+\left[\sum_{n=0}^{\infty} t^{n}\right]\left[\sum_{n=0}^{\infty} t^{n} \tilde{\psi}_{n}(A)\right]=\hat{\psi}(t, \bar{A})+\frac{1}{1-t} \hat{\psi}(t, A)
\end{aligned}
$$

when $|t|<1$, yielding (20) thanks to (21).

\section{Almost sure behaviour of $\nu_{n}$ when $\mathbb{S}=\mathbb{Z}$}

We assume in this section the branching random walk evolves on the lattice set $\mathbb{S}=\mathbb{Z}$, i.e.

$$
\mathbb{P}(\forall|x|=1, \tilde{V}(x) \in \mathbb{Z})=1,
$$

and that $\bar{A}$-i.e. the set on which the population reproduces- is finite. We also suppose that displacement $V$ is not 0 .

Under these assumptions, the individuals of the branching random walk

that stay in $\bar{A}$ reproduce according to a multitype Galton-Watson process, with mean $M$ matrix defined by

$$
\forall i, j \in \bar{A}, \quad M_{i, j}=\mathbb{E}_{i}\left[\sum_{|u|=1} \mathbb{1}_{\{V(u)=j\}}\right]=p\left[\delta_{i, j}+\mathbb{E}(N) \mathbb{P}(V=j-i)\right] .
$$

More precisely, an individual at position $i$ can be thought of as having type $i$. We refer to [2, Chapter 5] for the precise definition of multitype GaltonWatson processes.

One can check easily that $M$ is an irreducible matrix, as distribution of $V$ is not $\delta_{0}$ and is symmetric. Let $\rho(M)>0$ be the maximal eigenvalue of $M$. By Perron-Frobenius theory, there exists a unique left eigenvector $\mathbf{b}=\left(b_{j}, j \in \bar{A}\right)$ with positive entries, such that

$$
\forall j \in \bar{A}, \quad \sum_{i \in \bar{A}} b_{i} M_{i, j}=\rho(M) b_{j} \geq 0 \quad \text { and } \quad \sum_{j \in \bar{A}} b_{j}=1 .
$$


Observe that summing the first equation in $j$, we have

$$
\rho(M)=\sum_{i, j \in \bar{A}} b_{i} M_{i, j}=p\left(1+\mathbb{E}(N) \sum_{i \in \bar{A}} b_{i} \mathbb{P}(V+i \in \bar{A})\right) .
$$

Up to the end of the section, we assume that $\rho(M)>1$. Under this assumption, the multitype Galton-Watson process of mean matrix $M$ is supercritical (see e.g. [2, Chapter 5, Theorem 3.2]), meaning that the survival event

$$
S=\left\{\forall n \in \mathbb{N}, \nu_{n}^{(1)}(1)>0\right\},
$$

on which the process conserves active individuals forever, has positive probability of occurrence. If $\rho(M) \leq 1$, the process would die out almost surely therefore there would have only a finite amount of individuals produced. We obtain first moment estimates in a particular case in Section 5.2.

Note that $\nu_{n}^{(1)}(1) \longrightarrow_{n \rightarrow \infty}+\infty$ a.s. on $S$. We next set

$$
\forall i \in \mathbb{Z}, \quad a_{i}= \begin{cases}b_{i} & \text { if } \quad i \in \bar{A}, \\ p \mathbb{E}(N) \frac{\rho(M)}{\rho(M)-1} \sum_{j \in \bar{A}} b_{j} \mathbb{P}(V=i-j) & \text { if } \quad i \in A .\end{cases}
$$

Observing that $\sum_{i \in \mathbb{Z}} a_{i}=1+\frac{\rho(M)}{\rho(M)-1}(p(1+\mathbb{E}(N))-\rho(M))$, we therefore renormalize the $a_{i}$ 's and set

$$
\forall i \in \mathbb{Z}, \quad \bar{a}_{i}=\frac{a_{i}}{1+\frac{\rho(M)}{\rho(M)-1}(p(1+\mathbb{E}(N))-\rho(M))} .
$$

The asymptotic behaviour of $\nu_{n}$ on $S$ is given by the following theorem.

Theorem 6. If $\rho(M)>1$, we have

$$
\frac{\nu_{n}}{\nu_{n}(1)} \underset{n \rightarrow+\infty}{\rightsquigarrow} \sum_{i \in \mathbb{Z}} \bar{a}_{i} \delta_{i} \quad \text { on } S
$$

Moreover, under the additional assumption $\mathbb{E}(N \log (1+N))<+\infty$, we have

$$
\lim _{n \rightarrow+\infty} \rho(M)^{-n} \nu_{n}(1)=W \quad \text { a.s. }
$$

for some r.v. $W$ which is positive on $S$.

To prove this result, we first study the asymptotic behaviour of $\nu_{n}^{(1)}$. 
Lemma 7. If $\rho(M)>1$, we have

$$
\frac{\nu_{n}^{(1)}}{\nu_{n}^{(1)}(1)} \underset{\substack{n \rightarrow+\infty \\ n \rightarrow \bar{A}}}{\rightsquigarrow} b_{i} \delta_{i} \quad \text { on } S
$$

Moreover, under the additional assumption $\mathbb{E}(N \log (1+N))<+\infty$, we have

$$
\lim _{n \rightarrow+\infty} \rho(M)^{-n} \nu_{n}^{(1)}(1)=\tilde{W} \quad \text { a.s. }
$$

with $\tilde{W}>0$ on $S$.

Proof. This result is a direct consequence of [7]. Indeed, we observe that

$$
\forall n \geq 0, \forall j \in \bar{A}, Z_{n}(j)=\sum_{|x|=n} \mathbb{1}_{\{V(x)=j\}}
$$

defines a multi-type Galton-Watson process $\left\{\left(Z_{n}(j), j \in \bar{A}\right), n \in \mathbb{N}\right\}$, where type $j \in \bar{A}$ here corresponds to the position of the individual. This process is supercritical under the assumption $\rho(M)>1$, so that (30) is a consequence of [7, Theorem 2]. Now, since an individual of type $i$ will produce at most a number of individuals of type $j$ distributed as $N$, one has that $\mathbb{E}_{i}\left(Z_{1}(j) \log \left(1+Z_{1}(j)\right)\right) \leq \mathbb{E}(N \log (1+N))$, so that condition $\mathbb{E}(N \log (1+N))<+\infty$ implies (31) thanks to [7, Theorem 1].

Knowing the behaviour of the process in the active zone, we can deduce the behaviour in the passive zone.

Corollary 8. Using the same notations as in the previous lemma, if $\rho(M)>$ 1 then for any $j \in \mathbb{Z}$ the following limit holds

$$
\lim _{n \rightarrow+\infty} \frac{1}{\nu_{n}^{(1)}(1)} \sum_{|u|=n} \mathbb{1}_{\{V(u)=j\}}=a_{j} \quad \text { on } S .
$$

Proof. For any $j \in \mathbb{Z}$ and $n \geq 0$, let $Z_{n}(j)$ be defined as in (32). We observe that for any $i \in A$, we have

$$
Z_{n}(i)=\sum_{j \in \bar{A}} \sum_{k=1}^{n} \sum_{r=1}^{Z_{k}(j)} N_{r, k}^{j, i},
$$

where $N_{r, k}^{j, i}$ is the number of children made at position $i$ by the $r$ th individual alive at generation $k$ and position $j$, see Figure 2. We observe that $\left(N_{r, k}^{j, i}, r \geq\right.$ 


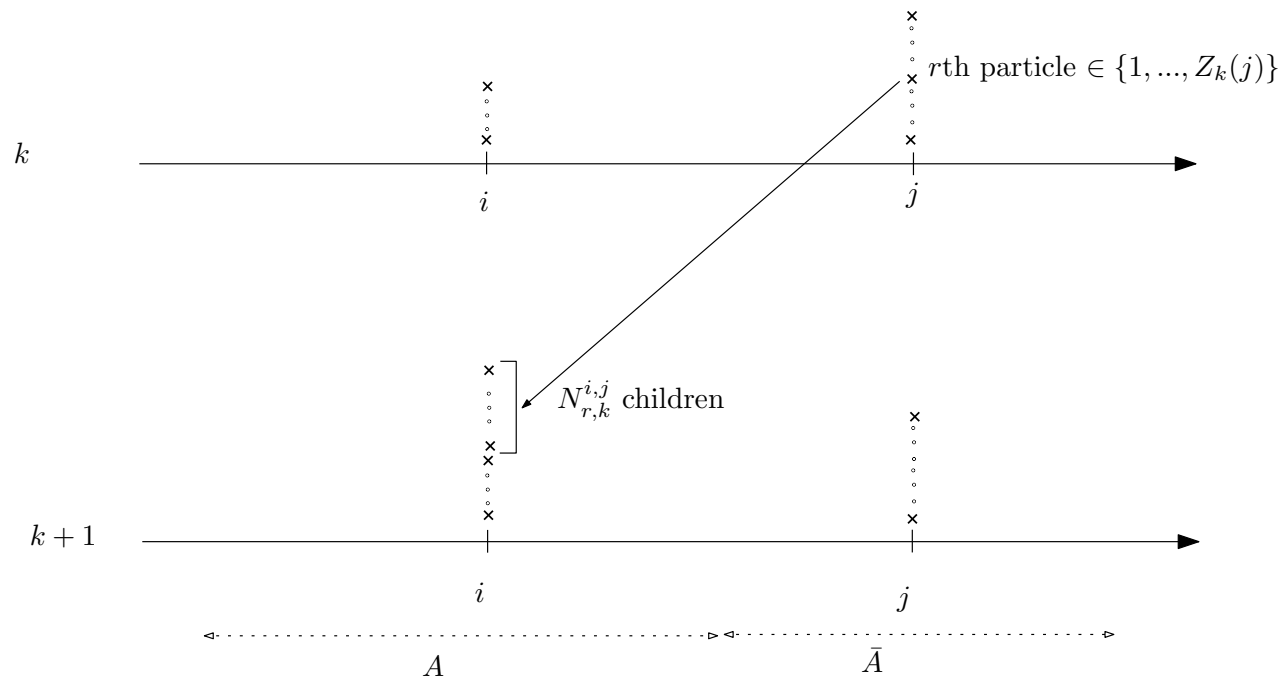

Figure 2: Branching random walk seen as a multitype Galton Watson process.

$1, k \geq 0)$ are i.i.d. random variables with mean $p \mathbb{E}(N) \mathbb{P}(V=j-i)$. Therefore, by the strong law of large numbers, we have, as $\sum_{k=1}^{n} Z_{k}(j) \longrightarrow_{n \rightarrow \infty}+\infty$ on $S$,

$$
\lim _{n \rightarrow+\infty} \frac{\sum_{k=1}^{n} \sum_{r=1}^{Z_{k}(j)} N_{r, k}^{j, i}}{\sum_{k=1}^{n} Z_{k}(j)}=p \mathbb{E}(N) \mathbb{P}(V=j-i) \quad \text { on } S .
$$

By (30), for any $j \in \bar{A}$, we have

$$
\lim _{n \rightarrow+\infty} \frac{Z_{n}(j)}{\nu_{n}^{(1)}(1)}=b_{j} \quad \text { on } S .
$$

Moreover, we observe that

$$
\lim _{n \rightarrow+\infty} \frac{\nu_{n+1}^{(1)}(1)}{\nu_{n}^{(1)}(1)}=\rho(M) \text { on } S,
$$

therefore, by the Stolz-Cesàro theorem we have

$$
\begin{aligned}
\lim _{n \rightarrow+\infty} \frac{1}{\nu_{n}^{(1)}(1)} \sum_{k=1}^{n} Z_{k}(j) & =\lim _{n \rightarrow+\infty} \frac{Z_{n+1}(j)}{\nu_{n+1}^{(1)}(1)-\nu_{n}^{(1)}(1)} \\
& =\lim _{n \rightarrow+\infty} \frac{Z_{n+1}(j)}{\nu_{n+1}^{(1)}(1)} \frac{\nu_{n+1}^{(1)}(1)}{\nu_{n+1}^{(1)}(1)-\nu_{n}^{(1)}(1)}=\frac{\rho(M) b_{j}}{\rho(M)-1} \quad \text { on } S .
\end{aligned}
$$


We conclude that

$$
\lim _{n \rightarrow+\infty} \frac{Z_{n}(i)}{\nu_{n}^{(1)}(1)}=\sum_{j \in \bar{A}} p \mathbb{E}(N) \mathbb{P}(V=j-i) \frac{b_{j}}{\rho(M)-1}=a_{i} \quad \text { on } S .
$$

We conclude by observing that Theorem 6 is a direct consequence of Lemma 7 and Corollary 8.

\section{Discrete set case and Rademacher distributed displacements}

We suppose in this section that $\mathbb{S}=\mathbb{Z}$, and that the complementary of the trapping zone is of the form

$$
\bar{A}=\{0, \ldots, L\}
$$

for some $L \in \mathbb{N}^{*}$, that initial position $a$ is in $\{0, \ldots, L\}$, and $V$ takes its values in $\mathbb{Z}$. We wish in this section to obtain explicit expressions of $\tilde{\psi}^{(\kappa)}(A)$, $\tilde{\psi}^{(\kappa)}(\bar{A})$ (for an adequate $\kappa$ ), the expression of the corresponding generating functions $\hat{\psi}(t, A)$ and $\hat{\psi}(t)$ as well as the limiting renormalized behaviour random measure $\nu_{n}$ as $n \rightarrow \infty$ in the case where survival occurs with positive probability. We recall that if the constant $\kappa$ is larger than 1 , we can obtain an expression of $\psi_{\kappa}$ using Formula (15).

In order to obtain $\tilde{\psi}^{(\kappa)}(A)$ and $\hat{\psi}(t, A)$, we have to first compute $\tilde{\psi}_{n}(A)$. Let us first note that, by Lemma 2, one has

$$
\tilde{\psi}_{n}(A)=m^{n} \mathbb{E}\left[e^{u S_{n}} \mathbb{1}_{\left\{S_{j} \in \bar{A}, \forall j=1, \ldots, n-1, S_{n} \in A\right\}}\right]=m^{n} \mathbb{E}\left[e^{u S_{n}} \mathbb{1}_{\left\{T_{\bar{A}}=n\right\}}\right]
$$

where $\left\{S_{n}=a+\sum_{k=1}^{n} \xi_{k}, n \geq 0\right\}$ is the associated $\mathbb{Z}$ valued random walk defined by (6), and where we recall that $T_{\bar{A}}$ is the exit time of this random walk out of $\bar{A}$. It is thus clear $\tilde{\psi}_{n}(A)$ is available if and only if the distribution of $T_{\bar{A}}$ jointly to the position of the random walk $S_{T_{\bar{A}}}$ on exiting this set is known. This joint distributions are in general not available, which is why we will restrict here to the case where displacements are Rademacher distributed, i.e.

$$
V \sim \frac{1}{2} \delta_{-1}+\frac{1}{2} \delta_{+1}
$$


in which case one might consider that $\mathbb{S}=\{-1, \ldots, L+1\}, A=\{-1, L+1\}$, $m(u)=p(1+\mathbb{E} N \cosh (u))$ and $I=\mathbb{R}$. One has from (34)

$$
\begin{aligned}
\tilde{\psi}_{n}(A) & =m^{n} \mathbb{E}\left[e^{u S_{n}} \mathbb{1}_{\left\{T_{\bar{A}}=n\right\}}\right] \\
& =m^{n} e^{u(L+1)} \mathbb{P}\left[T_{+}=n, T_{+}<T_{-}\right]+m^{n} e^{u .(-1)} \mathbb{P}\left[T_{-}=n, T_{-}<T_{+}\right],
\end{aligned}
$$

where $T_{-}$and $T_{+}$be the exit times (with values in $\mathbb{N} \cup\{+\infty\}$ ) of the one dimensional random walk $\left\{S_{n}, n \geq 0\right\}$ out of $\bar{A}$ through respectively 0 and $L$.

Remark 9. An example of distribution for $V$ for which $\mathbb{E}\left[e^{u S_{n}} \mathbb{1}_{\left\{T_{\bar{A}}=n\right\}}\right]$ is available is the case where displacements are bilateral geometric distributed, i.e. such that $V$ or $-V$ is $\mathcal{G}(p)$ distributed with probability $1 / 2$. In that case indeed, (35) is replaced by

$\tilde{\psi}_{n}(A)=m^{n} \mathbb{E}\left(e^{u(Y+L)}\right) \mathbb{P}\left[T_{+}=n, T_{+}<T_{-}\right]+m^{n} \mathbb{E}\left(e^{-u Y}\right) \mathbb{P}\left[T_{-}=n, T_{-}<T_{+}\right]$

where $Y \sim \mathcal{G}(p)$, by the memoryless distribution of the $\mathcal{G}(p)$ distribution. We will focus on the Rademacher distribution in what follows. It is however noted that similar results hold in the case of bilateral geometric distribution.

\subsection{Computations of $\psi^{(\kappa)}$.}

In the case of Rademacher displacements, random walk $S_{n}$ is equal to $a+\sum_{k=1}^{n} \xi_{k}$, where $\left(\xi_{k}\right)_{k \in \mathbb{N}}$ is i.i.d. with distribution thanks to (6), i.e. such that

$$
\xi_{k} \sim \frac{p}{m} \delta_{0}+\frac{p \mathbb{E} N}{2 m} \delta_{-1}+\frac{p \mathbb{E} N}{2 m} \delta_{+1}, \quad k \in \mathbb{N} .
$$

Looking back at (35), we need thus to give estimations on the two following quantities as $n \rightarrow+\infty$ :

$$
\begin{aligned}
\mathbb{P}\left[S_{j} \in \bar{A}, \forall j=1, \ldots, n-1, S_{n}=L+1\right] & =\mathbb{P}\left[T_{+}=n, T_{+}<T_{-}\right] \\
\mathbb{P}\left[S_{j} \in \bar{A}, \forall j=1, \ldots, n-1, S_{n}=-1\right] & =\mathbb{P}\left[T_{-}=n, T_{-}<T_{+}\right] .
\end{aligned}
$$

These two quantities correspond to the probability that a Markov chain $\left\{X_{n}, n \in \mathbb{N}\right\}$, starting from $X_{0}=a$, with values in $\{-1,0, \ldots, L, L+1\}$ and two absorbing states -1 and $L+1$ respectively reach $L+1$ and -1 
at time $n$ for the first time, and with transition probability $\left(Q_{i j}\right)_{i, j=-1, \ldots, L+1}$ defined by

$$
\begin{aligned}
& Q_{i, i-1}=Q_{i, i+1}=\frac{p \mathbb{E} N}{2 m}, \quad Q_{i, i}=\frac{p}{m}, \quad i=0, \ldots, L, \\
& Q_{-1,-1}=1, \quad Q_{L+1, L+1}=1 .
\end{aligned}
$$

Letting $\mathbf{e}_{i}$ the column vector of size $L+3$ such that its $i$ th component, $i \in\{-1, \ldots, L+1\}$, is 1 , and 0 otherwise. Since $L+1$ is absorbing, and since state $L+1$ is reachable only from state $L$, one has that

$$
\begin{aligned}
& \mathbb{P}\left[T_{+}=n, T_{+}<T_{-}\right]=\mathbb{P}\left[X_{n}=L+1, X_{n-1}=L\right]=\frac{p \mathbb{E} N}{2 m} \mathbf{e}_{a}^{\prime} Q^{n-1} \mathbf{e}_{L}, \\
& \mathbb{P}\left[T_{-}=n, T_{-}<T_{+}\right]=\mathbb{P}\left[X_{n}=-1, X_{n-1}=0\right]=\frac{p \mathbb{E} N}{2 m} \mathbf{e}_{a}^{\prime} Q^{n-1} \mathbf{e}_{0},
\end{aligned}
$$

where we remind that $a \in\{0, \ldots, L\}$ is the initial position of both the branching process and $\left\{X_{n}, n \in \mathbb{N}\right\}$, so that probabilities in (38) are equal to 0 when $n=0$. Letting $\tilde{Q}=\left(Q_{i, j}\right)_{i, j=0, \ldots, L}$ the $(L+1) \times(L+1)$ submatrix of $Q$ obtained by considering indices $0, \ldots, L$, it can be easily checked that $\left[Q^{n-1}\right]_{i, j}=\left[\tilde{Q}^{n-1}\right]_{i, j}$ for all $i, j$ in $\{0, \ldots, L\}$, so that one can write

$$
\begin{aligned}
\mathbf{e}_{x}^{\prime} Q^{n-1} \mathbf{e}_{L} & =\tilde{\mathbf{e}}_{a}^{\prime} \tilde{Q}^{n-1} \tilde{\mathbf{e}}_{L}, \\
\mathbf{e}_{x}^{\prime} Q^{n-1} \mathbf{e}_{0} & =\tilde{\mathbf{e}}_{a}^{\prime} \tilde{Q}^{n-1} \tilde{\mathbf{e}}_{0}
\end{aligned}
$$

where $\tilde{\mathbf{e}}_{i}, i=0, \ldots, L$, is the column vector of size $L+1$ of entry $i$ equal to 1 and 0 if not. Since $\tilde{Q}$ is an irreducible and non negative matrix, it admits a largest eigenvalue $\rho(Q)$ which is less than 1 since $\tilde{Q}$ is substochastic. Remember that $a$ is the starting point of the branching process as well as of Markov chain $\left\{X_{n}, n \in \mathbb{N}\right\}$. By the Perron Frobenius theory (see Theorem 8.2.9 p.499 of $[3])$, one has

$$
\left[\rho(Q)^{-1} \tilde{Q}\right]^{n} \longrightarrow \mathbf{x y}^{\prime}:=L_{Q}, \quad n \rightarrow+\infty
$$

where $\mathbf{x}$ and $\mathbf{y}$ are right and left eigenvectors associated to $\rho(Q)$ such that $\mathbf{x}^{\prime} \mathbf{y}=1$. From (38), (39) and (40), we thus obtain the following estimates for (36) and (37)

$$
\begin{aligned}
& \mathbb{P}\left[T_{+}=n, T_{+}<T_{-}\right] \sim \rho(Q)^{n-1} \frac{p \mathbb{E} N}{2 m} \tilde{\mathbf{e}}_{a}^{\prime} L_{Q} \tilde{\mathbf{e}}_{L}, \\
& \mathbb{P}\left[T_{-}=n, T_{-}<T_{+}\right] \sim \rho(Q)^{n-1} \frac{p \mathbb{E} N}{2 m} \tilde{\mathbf{e}}_{a}^{\prime} L_{Q} \tilde{\mathbf{e}}_{0},
\end{aligned}
$$

as $n \rightarrow+\infty$. 
We now compute $\rho(Q), \mathbf{x}$ and $\mathbf{y}$ explicitly, using the fact that $\mathbf{x}$ (and $\mathbf{y}$ ) are the unique right (and left) eigenvectors of $\tilde{Q}$ with only positive entry (up to a multiplicative constant), and $\rho(Q)$ is the associated eigenvalue. We set

$x_{j}=\sqrt{\frac{2}{L+1}} \sin \frac{\pi(j+1)}{L+2}, j=-1,0, \ldots, L+1, \quad \mathbf{x}=\left(x_{0}, \ldots, x_{L}\right)$ and $\mathbf{y}=\mathbf{x}^{\prime}$,

and we observe that for all $j \in\{0, \ldots, L\}$, we have

$$
\begin{aligned}
(\tilde{Q} x)_{j} & =x_{j} \frac{p}{m}+\frac{p \mathbb{E} N}{2 m}\left(x_{j-1}+x_{j+1}\right) \\
& =x_{j} \frac{p}{m}+\frac{p \mathbb{E} N}{2 m} \sqrt{\frac{2}{L+1}}\left(\sin \frac{\pi(j+2)}{L+2}+\sin \frac{\pi j}{L+2}\right) \\
& =x_{j} \frac{p}{m}+\frac{p \mathbb{E} N}{2 m} \sqrt{\frac{2}{L+1}}\left(2 \sin \frac{\pi(j+1)}{L+2} \cos \frac{\pi}{L+2}\right) \\
& =x_{j} \rho(Q),
\end{aligned}
$$

where we have set

$$
\rho(Q)=\frac{p}{m}\left(1+\mathbb{E} N \cos \frac{\pi}{L+2}\right) .
$$

With the same computations we obtain $\mathbf{y}^{\prime} \tilde{Q}=\rho(Q) \mathbf{y}^{\prime}$, and we observe easily that $\mathbf{x}^{\prime} \mathbf{y}=1$ with our choice of normalization.

Plugging these observations into (35) and using Proposition 4, we obtain the following result:

Proposition 10. One has, for an initial position $a \in\{0, \ldots, L\}$ that constant $\kappa$ such that $\tilde{\psi}_{n}(A) / \kappa^{n}$ converges (see (12)) is

$$
\kappa=m \rho(Q)
$$

where $\rho(Q)$ is given by (44). If $\kappa$ defined in (45) is larger than $1, \tilde{\psi}^{(\kappa)}(A)$ and $\psi^{(\kappa)}$ are given by

$$
\begin{aligned}
\tilde{\psi}^{(\kappa)}(A) & =\rho(Q)^{-1} \frac{p \mathbb{E} N}{2 m}\left[e^{u(L+1)} \tilde{\mathbf{e}}_{a}^{\prime} L_{Q} \tilde{\mathbf{e}}_{L}+e^{u \cdot(-1)} \tilde{\mathbf{e}}_{a}^{\prime} L_{Q} \tilde{\mathbf{e}}_{0}\right], \\
\psi^{(\kappa)} & =\left[\frac{m \rho(Q)}{m \rho(Q)-1}\right]\left[\frac{p(1+\mathbb{E} N \cosh (u))-1}{p(1+\mathbb{E} N \cosh (u))-m \rho(Q)}\right] \tilde{\psi}^{(\kappa)}(A) .
\end{aligned}
$$

Here matrix $L_{Q}$ is defined by (40).

Let us also note that Condition $\kappa<m$ required by Proposition 4 in order to get expression (47) is always fulfilled, as $\rho(Q)$ is always less than 1. 


\subsection{Asymptotic behaviour of expected number of particles in $A$ and $\bar{A}$.}

We recall that all expressions obtained previously depend on parameter $u \in I$. Let us consider the particular case when $u$ is equal to zero. To explicit this particular case, 0 is added as a superscript to the quantities $\psi, \psi_{1}, \psi_{2}$, $\tilde{\psi}_{k}(k \geq 1), \psi^{(\kappa)}$ and $\tilde{\psi}^{(\kappa)}$.

- $\lim _{n \rightarrow \infty} \psi_{n}^{0}$ represents the expected limit number of offsprings ;

- $\lim _{n \rightarrow \infty} \psi_{n}^{0(1)}$ represents the expected limit number of offsprings which are in $\bar{A}$ that is to say not in the trap zone ;

- $\lim _{n \rightarrow \infty} \psi_{n}^{0^{(2)}}$ represents the expected limit number of offsprings which are in the trap zone $A$.

Now, let us distinguish the three different cases $\kappa=m \rho(Q)>1, \kappa=$ $m \rho(Q)<1$ and $\kappa=m \rho(Q)=1$.

Case $\kappa=m \rho(Q)>1$. From (16),

$$
\begin{aligned}
& \lim _{n \rightarrow \infty} \frac{\psi_{n}^{0^{(1)}}}{\kappa^{n}}=\tilde{\psi}^{0^{(\kappa)}}(\bar{A}), \\
& \lim _{n \rightarrow \infty} \frac{\psi_{n}^{0^{(2)}}}{\kappa^{n}}=\frac{\kappa}{\kappa-1} \tilde{\psi}^{0}(\kappa)(A),
\end{aligned}
$$

where $\tilde{\psi}^{(\kappa)}(\bar{A})$ and $\tilde{\psi}^{(\kappa)}(A)$ are given by (14) and (46) with $u=0$. As a consequence, when $n$ goes to infinity, the expected limit number of offspring which are not in the trap zone is equivalent to $\kappa^{n} \tilde{\psi}^{(\kappa)}(\bar{A})$ and goes to infinity since $\kappa>1$ and the expected limit number of offspring in the gap zone is equivalent to $\left(\kappa^{n+1} /(1-\kappa)\right) \tilde{\psi}^{0}{ }^{(\kappa)}(A)$ which goes to infinity too. To end this case, $\mathbb{E}\left(\nu_{n}(1)\right)$ is equivalent to $\kappa^{n}\left(\tilde{\psi}^{(\kappa)}(\bar{A})+(\kappa /(1-\kappa)) \tilde{\psi}^{(\kappa)}(A)\right)$ which goes logically to infinity.

Case $\kappa=m \rho(Q)<1$. Let us first use Lemma 2 and write

$$
\begin{aligned}
\psi_{n}^{0(1)} & =\mathbb{E}_{a}\left[\sum_{|z|=n} \mathbb{1}_{T_{A}(z)>n}\right]=m^{n} \mathbb{E}\left[\mathbb{1}_{\left\{S_{j} \in \bar{A}, j \leq n\right\}}\right]=m^{n} \mathbb{P}\left(\min \left(T_{+}, T_{-}\right)>n\right) \\
& =m^{n} \sum_{k=n+1}^{+\infty} \mathbb{P}\left(\min \left(T_{+}, T_{-}\right)=k\right) .
\end{aligned}
$$


From (41) and (42), and since $\rho(Q)<1$, one gets the following equivalent:

$$
\begin{aligned}
\psi_{n}^{0(1)} & \sim m^{n} \sum_{k=n+1}^{+\infty} \rho(Q)^{k} \frac{p \mathbb{E} N}{2 m}\left(\tilde{\mathbf{e}}_{a}^{\prime} L_{Q} \tilde{\mathbf{e}}_{L}+\tilde{\mathbf{e}}_{a}^{\prime} L_{Q} \tilde{\mathbf{e}}_{0}\right), \quad n \rightarrow+\infty \\
& =m^{n} \rho(Q)^{n} \frac{\rho(Q)}{1-\rho(Q)} \frac{p \mathbb{E} N}{2 m}\left(\tilde{\mathbf{e}}_{a}^{\prime} L_{Q} \tilde{\mathbf{e}}_{L}+\tilde{\mathbf{e}}_{a}^{\prime} L_{Q} \tilde{\mathbf{e}}_{0}\right) \\
& =\kappa^{n} \frac{\rho(Q)}{1-\rho(Q)} \frac{p \mathbb{E} N}{2 m}\left(\tilde{\mathbf{e}}_{a}^{\prime} L_{Q} \tilde{\mathbf{e}}_{L}+\tilde{\mathbf{e}}_{a}^{\prime} L_{Q} \tilde{\mathbf{e}}_{0}\right)
\end{aligned}
$$

As a consequence, the expected limit number of offspring which are not in the trap zone goes to zero since $\kappa<1$.

For the second term $\psi_{n}^{0(2)}$, let us first recall that

$$
\psi_{n}^{0(2)}=\sum_{k=0}^{n} \tilde{\psi}_{k}^{0}(A) .
$$

Since $\tilde{\psi}_{k}^{0}(A) \underset{k \rightarrow \infty}{\sim} \kappa^{k} \tilde{\psi}^{0}{ }^{(\kappa)}$ then $\sum_{k=0}^{\infty} \tilde{\psi}^{0}{ }_{k}(A)$ is finite. Then, we deduce that the expected limit number of offspring in the gap zone is finite.

Case $\kappa=m \rho(Q)=1$. Note that computation (48) becomes

$$
\psi_{n}^{0(1)} \sim \frac{\rho(Q)}{1-\rho(Q)} \frac{p \mathbb{E} N}{2 m}\left(\tilde{\mathbf{e}}_{a}^{\prime} L_{Q} \tilde{\mathbf{e}}_{L}+\tilde{\mathbf{e}}_{a}^{\prime} L_{Q} \tilde{\mathbf{e}}_{0}\right), \quad n \rightarrow+\infty,
$$

so that the expected limit number of offspring which are not in the trap zone converges to a finite limit. As to $\psi_{n}^{0^{(2)}},(49)$ still holds but is this time divergent as $n \rightarrow \infty$.

Gathering all these cases, we thus have proved the following result:

Proposition 11. The mean number $\psi_{n}^{0^{(1)}}$ and $\psi_{n}^{(2)}$ of particles respectively in the trap zones $A$ and in the non-trap zone $\bar{A}$ evolve according to the following cases for $\kappa$ defined by (45), as $n \rightarrow+\infty$. 


\begin{tabular}{|c|c|}
\hline Supercritical case $\kappa>1$ & $\begin{aligned} \psi_{n}^{0^{(1)}} & \sim \kappa^{n} \tilde{\psi}^{(\kappa)}(\bar{A}) \longrightarrow+\infty \\
\psi_{n}^{0}(2) & \sim \kappa^{n+1} \frac{1}{1-\kappa} \tilde{\psi}^{(\kappa)}(A) \longrightarrow+\infty\end{aligned}$ \\
\hline Subcritical case $\kappa<1$ & $\begin{aligned} \psi_{n}^{0(1)} & \sim \kappa^{n} \frac{\rho(Q)}{1-\rho(Q)} \frac{p \mathbb{E} N}{2 m}\left(\tilde{\mathbf{e}}_{a}^{\prime} L_{Q} \tilde{\mathbf{e}}_{L}+\tilde{\mathbf{e}}_{a}^{\prime} L_{Q} \tilde{\mathbf{e}}_{0}\right) \longrightarrow 0 \\
\psi_{n}^{0}(2) & \sim \sum_{k=0}^{\infty} \tilde{\psi}_{k}(A)<+\infty\end{aligned}$ \\
\hline Critical case $\kappa=1$ & $\begin{aligned} & \psi_{n}^{0^{(1)}} \sim \frac{\rho(Q)}{1-\rho(Q)} \frac{p \mathbb{E} N}{2 m}\left(\tilde{\mathbf{e}}_{a}^{\prime} L_{Q} \tilde{\mathbf{e}}_{L}+\tilde{\mathbf{e}}_{a}^{\prime} L_{Q} \tilde{\mathbf{e}}_{0}\right)<+\infty \\
& \psi_{n}^{0(2)} \sim \sum_{k=0}^{n} \tilde{\psi}_{k}(A) \longrightarrow+\infty\end{aligned}$ \\
\hline
\end{tabular}

Here $\tilde{\psi}^{(\kappa)}(\bar{A})$ and $\tilde{\psi}^{(\kappa)}(A)$ are given by (14) and (46) with $u=0$.

\subsection{Generating function}

The generating function $\hat{\psi}(t)$ of of $\left(\psi_{n}\right)_{n \in \mathbb{N}}$ is provided in the following result.

Proposition 12. One has the following expressions:

$$
\begin{aligned}
\hat{\psi}(t, A) & =\frac{p \mathbb{E} N}{2} \mathbf{e}_{a}^{\prime}(I-t m Q)^{-1} \cdot\left[e^{u(L+1)} \mathbf{e}_{L}+e^{-u} \mathbf{e}_{0}\right], \\
\hat{\psi}(t) & =\frac{\frac{p \mathbb{E} N}{2} \mathbf{e}_{a}^{\prime}(I-t m Q)^{-1} \cdot\left[e^{u(L+1)} \mathbf{e}_{L}+e^{-u} \mathbf{e}_{0}\right]-e^{u a}}{m(u) t-1} \\
& +\frac{1}{1-t} \frac{p \mathbb{E} N}{2} \mathbf{e}_{a}^{\prime}(I-t m Q)^{-1} \cdot\left[e^{u(L+1)} \mathbf{e}_{L}+e^{-u} \mathbf{e}_{0}\right]
\end{aligned}
$$

for $|t|$ small enough and less than $1 / m(u)=1 /[p(1+\mathbb{E} N \cosh (u))]$, where $I$ is the identity matrix.

Proof. Expression of $\hat{\psi}(t, A)$ is obtained thanks to (38), (36) and (37) plugged into (35) in the definition $\hat{\psi}(t, A)=\sum_{n=0}^{\infty} t^{n} \tilde{\psi}_{n}(A)$ of the generating function. Since this involves summing $\sum_{n=0}^{\infty}(t m Q)^{n}$, the series converges when $t$ is small enough such that matrix $I-t m Q$ is invertible, i.e. $|t|<1 / \operatorname{mr}(Q)$ where $r(Q)$ is the largest eigenvalue in modulus of $Q$, which here is equal to 1 as $Q$ is the transition matrix of a Markov chain with absorbing states. Expression of $\hat{\psi}(t)$ is obtained thanks to Expression (20) in Proposition 5. We note that, since (20) is valid for $|t|<1 / m(u)$ and $m(u) \geq m$, the condition for both $I-t m Q$ being invertible and $\hat{\psi}(t)$ to be valid is $|t|<1 / m(u)$. 
5.4. Almost sure behaviour of $\nu_{n}$ in the supercritical case $\kappa>1$

We apply in this section the results developed in Section 4 to compute the almost sure behaviour of $\nu_{n}$. We observe that in this case matrix $M$ defined in (23) has the following expression:

$$
\forall i, j \in\{0, \ldots, L\}, \quad M_{i, j}=p \delta_{i, j}+\frac{p \mathbb{E} N}{2}\left(\delta_{i, j+1}+\delta_{i, j-1}\right) .
$$

We note, with the notation in Section 4 , that $M=m \tilde{Q}$, so that expression (44) in Subsection 5.1 immediately yields that $\rho(M)=\kappa$, and, inspired by (43), that eigenvector $\mathbf{b}$ and eigenvalue $\rho(M)$ are given by

$$
\begin{aligned}
\forall i \in \bar{A}=\{0, \ldots, L\}, \quad b_{j}=\frac{\sin (\pi /(L+2))}{\cos (\pi /(L+2))} \sin \frac{\pi(j+1)}{L+2} \\
\text { and } \quad \rho(M)=m \rho(Q)=p\left(1+\mathbb{E} N \cos \frac{\pi}{L+2}\right)=\kappa .
\end{aligned}
$$

In particular, the process survives with positive probability as soon as we are in the supercritical case

$$
\kappa>1 \quad \Longleftrightarrow \quad \cos \frac{\pi}{L+2}>\frac{1-p}{p \mathbb{E} N},
$$

that we assume is true in this section. Moreover, we have immediately from (26)

$$
\begin{aligned}
a_{-1} & =a_{L+1}=p \mathbb{E} N \frac{\kappa}{\kappa-1} \frac{\sin (\pi /(L+2))^{2}}{\cos (\pi /(L+2))}, \\
a_{i} & =b_{i}, \quad i=0, \ldots, L .
\end{aligned}
$$

We conclude from Theorem 6 the following result:

Proposition 13. Under condition (50), there exists a non negative random variable $W$ such that the following convergences occur:

$$
\begin{aligned}
& \frac{\nu_{n}}{\nu_{n}(1)} \underset{n \rightarrow+\infty}{\rightsquigarrow} \sum_{i=-1}^{L+1} \bar{a}_{i} \delta_{i} \quad \text { on } S, \\
& \kappa^{-n} \nu_{n} \underset{n \rightarrow+\infty}{\rightsquigarrow} W \sum_{j=-1}^{L+1} a_{j} \delta_{j} \quad \text { a.s. }
\end{aligned}
$$

where $S$ is the set of survival of the branching random walk, and $\bar{a}_{i}$ is defined by (27) and $a_{-1}, \ldots, a_{L+1}$ are given by (51). 


\section{Laplace distributed displacements}

We suppose in this section that $\mathbb{S}=\mathbb{R}$, the active zone $\bar{A}$ is the open bounded interval

$$
\bar{A}=(-1,1),
$$

and that displacements are Laplace distributed with parameter $\mu$, i.e. positive or negative with probability $1 / 2$, with exponential distribution in absolute value with parameter $\mu$ (denoted by $V \sim \mathcal{L}(\mu)$ in the following).

We are here interested in the generating function $\hat{\psi}(t)$ of $\left(\psi_{n}\right)_{n \in \mathbb{N}}$ as defined in (4). As in (35), we wish to use Lemma 2, yielding

$$
\tilde{\psi}_{n}(A)=m^{n} \mathbb{E}\left[e^{u S_{n}} \mathbb{1}_{\left\{S_{j} \in \bar{A}, \forall j=1, \ldots, n-1, S_{n} \in A\right\}}\right] .
$$

The corresponding random walk $\left\{S_{n}=a+\sum_{k=1}^{n} \xi_{k}, n \geq 0\right\}$ is defined thanks to (6), with distribution

$$
\xi_{k} \sim \frac{p}{m} \delta_{0}+\frac{p \mathbb{E} N}{m} \mathcal{L}(\mu), \quad k \in \mathbb{N} .
$$

Since overshooting +1 (resp. undershooting -1) for the random walk $\left\{S_{n}, n \geq\right.$ $0\}$ necessarily corresponds to an exponentially distributed jump, one has from the memoryless property that

$$
\begin{aligned}
\tilde{\psi}_{n}(A) & =m^{n} \mathbb{E}\left[e^{u S_{n}} \mathbb{1}_{\left\{S_{j} \in \bar{A}, \forall j=1, \ldots, n-1, S_{n}<-1\right\}}\right]+m^{n} \mathbb{E}\left[e^{u S_{n}} \mathbb{1}_{\left\{S_{j} \in \bar{A}, \forall j=1, \ldots, n-1, S_{n}>1\right\}}\right] \\
& =m^{n} e^{-u} \frac{\mu}{\mu-u} \mathbb{P}\left[S_{j} \in \bar{A}, \forall j=1, \ldots, n-1, S_{n}<-1\right] \\
& +m^{n} e^{u} \frac{\mu}{\mu+u} \mathbb{P}\left[S_{j} \in \bar{A}, \forall j=1, \ldots, n-1, S_{n}>1\right]
\end{aligned}
$$

where we suppose that $|u|<\mu$. Both probabilities on the right-hand side of (54) correspond to the distribution of the exit time of $\left\{S_{n}, n \geq 0\right\}$ out of $\bar{A}$ and can be written as (similarly to (36)-(37))

$$
\begin{gathered}
\mathbb{P}\left[S_{j} \in \bar{A}, \forall j=1, \ldots, n-1, S_{n}<-1\right]=\mathbb{P}\left[T_{-1}=n, T_{-1}<T_{1}\right], \\
\mathbb{P}\left[S_{j} \in \bar{A}, \forall j=1, \ldots, n-1, S_{n}>1\right]=\mathbb{P}\left[T_{1}=n, T_{1}<T_{-1}\right],
\end{gathered}
$$

for all $n \in \mathbb{N}$, where $T_{-1}\left(\right.$ resp. $\left.T_{1}\right)$ represents the first instant when $\left\{S_{n}, n \geq\right.$ $0\}$ downcrosses -1 (resp. upcrosses +1 ), and $T:=\min \left(T_{-1}, T_{1}\right)$ is the first exit time out of $\bar{A}$. 
From (54), (55) and (56) one obtains the following expression for $\hat{\psi}(t, A)$

$$
\hat{\psi}(t, A)=e^{-u} \frac{\mu}{\mu-u} \phi_{-}(t m)+e^{u} \frac{\mu}{\mu+u} \phi_{+}(t m)
$$

where $\phi_{-}$and $\phi_{+}$are Laplace transforms of $T_{-1}$ and $T_{1}$ on the event that first exit from $\bar{A}$ is through -1 and +1 , i.e.

$$
\phi_{-}(t):=\mathbb{E}\left[t^{T_{-1}} \mathbb{1}_{\left\{T_{-1}<T_{1}\right\}}\right], \quad \phi_{+}(t):=\mathbb{E}\left[t^{T_{1}} \mathbb{1}_{\left\{T_{1}<T_{-1}\right\}}\right], \quad|t|<1 .
$$

In the following we will make use of results from [4] that gives the expressions of such quantities $\phi_{-}(t)$ and $\phi_{+}(t)$ only when the increments of the random walk $\left\{S_{n}, n \geq 0\right\}$ are non lattice. Since this is not the case here (as seen in (53)), we will need the following lemma:

Lemma 14. Let $T_{-1}^{0}, T_{1}^{0}$ be the downcrossing and upcrossing times through -1 and +1 of random walk $\left\{S_{n}^{0}=\sum_{k=1}^{n} \xi_{k}^{0}, n \geq 0\right\}$ where $\xi_{k}^{0} \sim \mathcal{L}(\mu)$, and let

$$
\phi_{-}^{0}(t):=\mathbb{E}\left[t^{T_{-1}^{0}} \mathbb{1}_{\left\{T_{-1}^{0}<T_{1}^{0}\right\}}\right], \quad \phi_{+}^{0}(t):=\mathbb{E}\left[t^{T_{1}^{0}} \mathbb{1}_{\left\{T_{1}^{0}<T_{-1}^{0}\right\}}\right], \quad|t|<1,
$$

be the corresponding generating functions. $\phi_{-}(t)$ and $\phi_{+}(t)$ are given in function of $\phi_{-}^{0}(t)$ and $\phi_{+}^{0}(t)$ by

$$
\phi_{ \pm}(t)=\frac{1}{1-t p \mathbb{E}(N) / m} \phi_{ \pm}^{0}\left(\frac{-t p}{m-t p \mathbb{E}(N)}\right), \quad|t|<m / p \mathbb{E}(N) .
$$

From the above result we obtain the following expression for $\hat{\psi}(t, A)$ and $\hat{\psi}(t)$ :

Proposition 15. Let us define the following invertible matrix

$$
M_{t}:=\left[\begin{array}{cc}
\frac{e^{\mu \sqrt{1-t}}}{1-\sqrt{1-t}} & \frac{e^{-\mu \sqrt{1-t}}}{1+\sqrt{1-t}} \\
\frac{e^{-\mu \sqrt{1-t}}}{1+\sqrt{1-t}} & \frac{e^{\mu \sqrt{1-t}}}{1-\sqrt{1-t}}
\end{array}\right], \quad 0<|t|<1,
$$

and let $\left(c_{-}(t), d_{-}(t)\right),\left(c_{+}(t), d_{+}(t)\right)$, be defined by

$$
\left(c_{-}(t), d_{-}(t)\right)^{\prime}=M_{t}^{-1}(1,0)^{\prime}, \quad\left(c_{+}(t), d_{+}(t)\right)^{\prime}=M_{t}^{-1}(0,1)^{\prime} .
$$


Then $\phi_{ \pm}^{0}$ have the following expressions

$$
\begin{aligned}
& \phi_{-}^{0}(t)=c_{-}(t) e^{-a \mu \sqrt{1-t}}+d_{-}(t) e^{a \mu \sqrt{1-t}}, \quad 0<|t|<1 . \\
& \phi_{+}^{0}(t)=c_{+}(t) e^{-a \mu \sqrt{1-t}}+d_{+}(t) e^{a \mu \sqrt{1-t}},
\end{aligned}
$$

Finally, the generating function of $\left(\tilde{\psi}_{n}(A)\right)_{n \in \mathbb{N}}$ is given by

$$
\begin{aligned}
& \hat{\psi}(t, A)=e^{-u} \frac{\mu}{\mu-u} \frac{1}{1-t p \mathbb{E}(N)} \phi_{-}^{0}\left(\frac{-t p}{1-t p \mathbb{E}(N)}\right) \\
& \quad+e^{u} \frac{\mu}{\mu+u} \frac{1}{1-t p \mathbb{E}(N)} \phi_{+}^{0}\left(\frac{-t p}{1-t p \mathbb{E}(N)}\right),
\end{aligned}
$$

for $|t|<1$, and $\hat{\psi}(t)$ is obtained thanks to (20) as well as the above expression for $\hat{\psi}(t, A)$.

Proof of Proposition 15. One just needs to be able to compute expressions of $\phi_{-}^{0}(t)$ and $\phi_{+}^{0}(t)$ required in Lemma 14 then plug (59) into (57) in order to obtain (62). This is done by using Theorem 5 from [4]. Indeed, $\xi_{k}^{0}$ defined in Lemma 14 is non lattice so that the latter result is applicable. Following [4], one has first to solve the so-called Lundberg equation (see Eq. (26) therein) which here takes the form

$$
\frac{1}{2} \frac{\mu}{\mu+z}+\frac{1}{2} \frac{\mu}{\mu-z}=\frac{2}{t}
$$

for a fixed $t \in(-1,1) \backslash\{0\}$ and of which solutions are $z= \pm \mu \sqrt{1-t}$. It is then not hard to check that $\left(c_{-}(t), d_{-}(t)\right),\left(c_{+}(t), d_{+}(t)\right)$ in $(60)$ are exactly the solutions to the linear system of equations following (27) in [4], and that Expressions (61) are exactly (27) from [4], where constants $(K, L)$ in this paper are respectively $(1,0)$ then $(0,1)$.

Proof of Lemma 14. First note that $\left\{S_{n}^{0}=\sum_{k=1}^{n} \xi_{k}^{0}, n \geq 0\right\}$ has same distribution as $\left\{S_{n}=\sum_{k=1}^{n} \xi_{k}, n \geq 0\right\}$ conditioned not to have zero increments. The idea of the proof is to intuitively consider that $T_{-1}$ and $T_{-1}^{0}$ only differ by a (random) number of times that the random walk $\left\{S_{n}=\sum_{k=1}^{n} \xi_{k}, n \geq 0\right\}$ had zero increments. It may be convenient to write

$$
\left(S_{n}\right)_{n \in \mathbb{N}} \stackrel{\mathcal{D}}{=}\left(\sum_{k=0}^{n} \Theta_{k} \xi_{k}^{0}\right)_{n \in \mathbb{N}}
$$


where $\left(\Theta_{k}\right)_{k \in \mathbb{N}}$ is an i.i.d. $\mathcal{B}(p \mathbb{E}(N) / m)$ distributed sequence of random variables, independent from the $\left(\xi_{k}^{0}\right)_{k \in \mathbb{N}}$. Let us introduce the following notation:

$$
p_{-}(j):=\mathbb{P}\left[T_{-1}^{0}=j, T_{-1}^{0}<T_{1}^{0}\right], \quad j \in \mathbb{N} .
$$

One then has from (63) that, for all $n \in \mathbb{N}$,

$$
\begin{aligned}
\mathbb{P}\left[T_{-1}=n, T_{-1}<T_{1}\right] & =\mathbb{E}\left[\mathbb{P}\left[T_{-1}=n, T_{-1}<T_{1} \mid \Theta_{1}, \ldots, \Theta_{n}\right]\right] \\
& =\mathbb{E}\left[p_{-}\left(n-\sum_{k=1}^{n} \Theta_{k}\right)\right] \\
& =\sum_{j=0}^{n} p_{-}(n-j)\left(\begin{array}{c}
n \\
j
\end{array}\right)\left(\frac{p \mathbb{E}(N)}{m}\right)^{j}\left(\frac{p}{m}\right)^{n-j} .
\end{aligned}
$$

One then computes

$$
\begin{aligned}
\phi_{-}(t) & =\sum_{n=0}^{\infty} t^{n} \mathbb{P}\left[T_{-1}=n, T_{-1}<T_{1}\right] \\
& =\sum_{n=0}^{\infty} t^{n} \sum_{j=0}^{n} p_{-}(n-j)\left(\begin{array}{c}
n \\
j
\end{array}\right)\left(\frac{p \mathbb{E}(N)}{m}\right)^{j}\left(\frac{p}{m}\right)^{n-j} \text { from (64), } \\
& =\sum_{j=0}^{\infty} \sum_{n \geq j} t^{n} p_{-}(n-j) \frac{n !}{(n-j) ! j !}\left(\frac{p \mathbb{E}(N)}{m}\right)^{j}\left(\frac{p}{m}\right)^{n-j} \\
& =\sum_{j=0}^{\infty} \sum_{n=0}^{\infty} t^{n+j} p_{-}(n) \frac{(n+j) !}{n ! j !}\left(\frac{p \mathbb{E}(N)}{m}\right)^{j}\left(\frac{p}{m}\right)^{n} \\
& =\sum_{n=0}^{\infty} t^{n}\left(\frac{p}{m}\right)^{n} p_{-}(n) \frac{1}{n !}\left[\sum_{j=0}^{\infty} \frac{(n+j) !}{j !}\left(\frac{t p \mathbb{E}(N)}{m}\right)^{j}\right]
\end{aligned}
$$

One has that $\sum_{j=0}^{\infty} \frac{(n+j) !}{j !} x^{j}=\sum_{j=0}^{\infty}(n+j) \ldots(j+1) x^{j}$ is the $n$th derivative of $x \mapsto \sum_{j=0}^{\infty} x^{j}=1 /(1-x),|x|<1$, i.e.

$$
\sum_{j=0}^{\infty} \frac{(n+j) !}{j !} x^{j}=(-1)^{n} n !(1-x)^{-n-1}
$$


so that in (65) we get, for $|t p \mathbb{E}(N) / m|<1$,

$$
\begin{aligned}
\phi_{-}(t) & =\sum_{n=0}^{\infty} t^{n}\left(\frac{p}{m}\right)^{n} p_{-}(n)(-1)^{n}\left(1-\frac{t p \mathbb{E}(N)}{m}\right)^{-n-1} \\
& =\frac{1}{1-t p \mathbb{E}(N) / m} \sum_{n=0}^{\infty} p_{-}(n)\left(\frac{-t p}{m-t p \mathbb{E}(N)}\right)^{n}
\end{aligned}
$$

which is exactly (59) for $\phi_{-}(t)\left(\phi_{+}(t)\right.$ is obtained similarly), for $t$ small enough such that $|t p \mathbb{E}(N) / m|<1$.

Remark 16. The special distribution $\mathcal{L}(\mu)$ for displacements was studied as a special case of the distribution which admit a rational Laplace transform. This class of such distributions includes the class of double sided PH-type distribution, i.e. $V=\Delta X$ where $\Delta$ is Rademacher distributed and $X$ is PH-type distributed. A closer look at the proofs shows that one of the main ingredients for obtaining the expression of the generating function (62) in Proposition 15 is to be able to get the distribution of the exit time of random walk $\left\{S_{n}, n \geq \mathbb{N}\right\}$ jointly to the overshoot or undershoot out of interval $\bar{A}=(-1,1)$, see (54) where we used the memoryless property of the exponential distribution. This joint distribution is actually given in [4] in the case of PH distributions (instead of the exponential distribution here), so that Proposition 15 could be adapted to include this type of distribution.

\section{The torus case}

We now suppose that $\mathbb{S}$ is the torus $\mathbb{T}$ with length of size 2, i.e. interval $[0,2)$ where we identify endpoints 0 and 2 . In that case, expression of $\nu_{n}\left(e^{u \cdot}\right)$ is given by

$$
\begin{aligned}
\nu_{n}\left(e^{u \cdot}\right):=\sum_{|x|=n} e^{u(V(x)-2\lfloor V(x) / 2\rfloor)} & =\sum_{|x|=n} e^{u \bar{V}(x)}, \\
\bar{V}(x) & :=V(x)-2\lfloor V(x) / 2\rfloor=V(x) \bmod 2,
\end{aligned}
$$

where $\lfloor z\rfloor$ stands for the integer part of $z \in \mathbb{R}$, for a branching random walk $(V(x), x \in \mathbf{T})$ on $\mathbb{R}$ with displacement distributed as $V$, so that $(\bar{V}(x), x \in$ $\mathbf{T})$ is a branching random walk on $\mathbb{T}$ with same displacement distribution. We suppose here that we are in the case $m=p(1+\mathbb{E} N)>1$. We start by studying the case where $\mathbb{T}$ does not contain any trapping zone. 
Theorem 17 (No trapping zone case). Let us suppose that $\mathbb{E}(N \log (N+$ $1))<+\infty$, and let $S$ be the set of non extinction. For any non lattice distribution of displacement $V$, one has the following a.s. convergence of empirical measure

$$
\frac{\nu_{n}}{\nu_{n}(1)} \underset{n \rightarrow+\infty}{\rightsquigarrow} \mathcal{U}(\mathbb{T}) \text { on } S,
$$

where $\mathcal{U}(\mathbb{T})$ is the uniform distribution on torus $\mathbb{T}$.

Proof. For $k \in \mathbb{Z}$ and $n \in \mathbb{N}$ the $k$ th Fourier coefficient of random measure $\nu_{n}$ is given by

$$
M_{n}(k):=\nu_{n}\left(e^{i k \pi \cdot}\right)=\int_{\mathbb{T}} e^{i k \pi x} \nu_{n}(d x),
$$

One verifies that $M_{n}(k)=\sum_{|u|=n} e^{i \pi k V(u)}$, i.e. is the Fourier coefficient of the branching random walk $(V(x), x \in \mathbf{T})$ on $\mathbb{R}$. We write $\phi_{k}:=\mathbb{E}\left(M_{1}(k)\right)=$ $p\left(1+\mathbb{E}(N) \mathbb{E}\left(e^{i \pi k V}\right)\right)$. Observe that for any $k \neq 0$, and because $V$ is non lattice,

$$
\left|\phi_{k}\right|=p\left|1+\mathbb{E}(N) \mathbb{E}\left(e^{i k \pi V}\right)\right|<p(1+\mathbb{E} N)=m .
$$

By [5, Theorem 1], $\left(\frac{M_{n}(k)}{\phi_{k}^{n}}\right)_{n \in \mathbb{N}}$ is a martingale. Consequently, and because of the above strict inequality, $\mathbb{E}\left(\sum_{n=0}^{\infty} \frac{M_{n}(k)}{m^{n}}\right)$ is finite for $k \neq 0$ and thus $\frac{M_{n}(k)}{m^{n}}$ converges a.s. to 0 for any $k \neq 0$. Moreover, by classical Galton-Watson theory, $M_{n}(0) / m^{n} \rightarrow Z$ a.s. for some r.v. $Z \geq 0$, such that $Z=0$ is the set of extinction $S$ under the assumption $\mathbb{E}\left(M_{n}(0) \ln \left(M_{n}(0)+1\right)\right)<+\infty$, which one can check is equivalent to condition $\mathbb{E}(N \log (N+1))<+\infty$. We deduce that for any measurable bounded function $f: \mathbb{T} \rightarrow \mathbb{R}$,

$$
\lim _{n \rightarrow+\infty} \frac{\nu_{n}(f)}{m^{n}}=Z \int_{0}^{2} f(s) d s
$$

As a result, $\nu_{n} / \nu_{n}(1)$ converges on $S$ towards the uniform distribution on the torus.

We next suppose that $\mathbb{T}$ admits a trapping zone $[1,2)$ and a non trapping zone $[0,1)$. This is equivalent to consider a branching random walk $(V(x), x \in \mathbf{T})$ on $\mathbb{R}$ with displacement distributed as $V$, and trapping zone

$$
A:=\cup_{j \in \mathbb{Z}} A_{j}, \quad A_{j}:=[2 j+1,2 j+2)=A+2 j .
$$

The non trapping zone is then $\bar{A}$ such that 

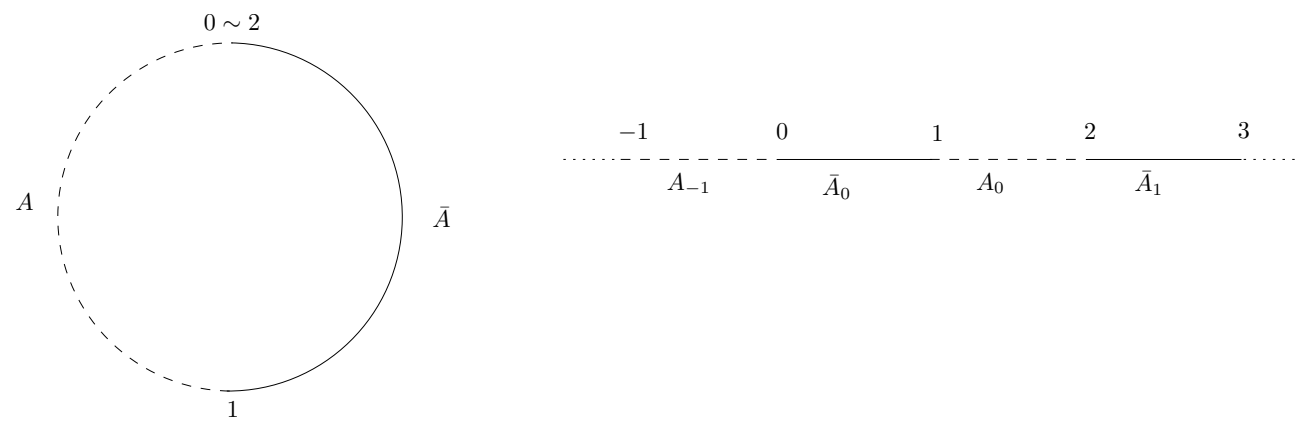

Figure 3: Trapping zone on torus.

$$
\bar{A}:=\cup_{j \in \mathbb{Z}} \bar{A}_{j}, \quad \bar{A}_{j}:=[2 j, 2 j+1)=\bar{A}+2 j,
$$

see Figure 3. We will also consider from now that displacements are, as in Section 6, Laplace distributed, namely $V \sim \mathcal{L}(\mu)$ for some $\mu>0$. Following the $\mathbb{S}=\mathbb{R}$ case studied in Section 6 , we are interested in

$$
\hat{\psi}(t, a):=\sum_{n=0}^{\infty} t^{n} \mathbb{E}_{a}\left(\nu_{n}\left(e^{u \cdot}\right)\right) .
$$

where $a \in \bar{A}_{0}$ is the initial position of the first particle, for a $u \in \mathbb{R}$ fixed throughout this section. We are interested in an integral equation satisfied by $\hat{\psi}(t,$.$) . Since displacements have same distribution as in Section 6$, we briefly recall some notation. We introduce and recall again the associated random walk $\left(S_{n}\right)_{n \in \mathbb{N}}$ defined in Lemma 2 by (6), with here the $\xi_{n}$ 's defined as in (53), i.e.

$$
\xi_{k} \sim \frac{p}{m} \delta_{0}+\frac{p \mathbb{E} N}{m} \mathcal{L}(\mu), \quad k \in \mathbb{N} .
$$

Furthermore, we recall the notation given in Lemma 14

$$
\phi_{ \pm}(t)=\phi_{ \pm, a}(t)=\frac{1}{1-t p \mathbb{E}(N) / m} \phi_{ \pm, a}^{0}\left(\frac{-t p}{m-t p \mathbb{E}(N)}\right), \quad|t|<m / p \mathbb{E}(N),
$$

stressing the dependence on the initial position $a$, where $\phi_{+, a}^{0}(z)$ and $\phi_{+, a}^{0}(z)$ are the generating functions respectively of the left and right exit times of random walk $\left(S_{n}\right)_{n \in \mathbb{N}}$ out of $\bar{A}_{0}=[0,1)$ starting from $a \in \bar{A}_{0}$, given by (61). It is not difficult to obtain an expression for these quantities, as in 
Proposition 15, so we will consider that those quantities $\phi_{ \pm, a}^{0}(z), a \in \bar{A}_{0}$, are available.

The main result of this section is the following theorem.

Theorem 18. Let us suppose that the trapping zone is as in Figure 3 and $V \sim \mathcal{L}(\mu)$. Then $a \in \bar{A}_{0} \mapsto \hat{\psi}(t, a)$ is the unique continuous solution to the following equation:

$$
\begin{aligned}
\hat{\psi}(t, a) & =\hat{\psi}_{a}\left(t, \bar{A}_{0}\right)+\hat{\psi}_{a}(t, A)+\int_{\bar{A}_{0}} \hat{\psi}(t, v) \mu e^{-\mu v} d v \frac{e^{-\mu}}{1-e^{-2 \mu}} \phi_{+}(m t) \\
& +\int_{\bar{A}_{0}} \hat{\psi}(t, v) \mu e^{\mu v} d v \frac{e^{-2 \mu}}{1-e^{-2 \mu}} \phi_{-}(m t)
\end{aligned}
$$

where $t$ and $u$ and small enough such that $(t, u)$ belong to set

$$
\left\{(s, v) \in \mathbb{R}^{2}|| v|<\mu,| s \mid<\frac{p}{m}+\frac{p \mathbb{E}(N)}{m} \frac{\mu^{2}}{\mu^{2}-v^{2}}\right\}
$$

and where $\hat{\psi}_{a}\left(t, \bar{A}_{0}\right), \hat{\psi}_{a}(t, A)$ are given by

$$
\begin{aligned}
\hat{\psi}_{a}\left(t, \bar{A}_{0}\right):= & \frac{\mu^{2}-u^{2}}{\mu^{2}-u^{2}-t\left(p\left(\mu^{2}-u^{2}\right)+p \mathbb{E}(N) \mu^{2}\right)} \\
& \cdot\left[e^{u a}-e^{u} \frac{\mu}{\mu-u} \phi_{+}(t m)-\frac{\mu}{\mu+u} \phi_{-}(t m)\right], \\
\hat{\psi}_{a}(t, A):= & \frac{1}{1+e^{-\mu}} \phi_{-}(t m)+e^{u} \frac{e^{-\mu}}{1+e^{-\mu}} \phi_{+}(t m) .
\end{aligned}
$$

The remaining of this section is dedicated to proving Theorem 18. We first establish that $\hat{\psi}(t,$.$) is solution to integral equation (68). Then we prove$ that such a solution is unique among the set of continuous functions from $\bar{A}_{0}$ to $\mathbb{R}$.

Establishing Equation (68). As in (9), we split $\mathbb{E}_{a}\left(\nu_{n}\left(e^{u \cdot}\right)\right)$ in three terms, according to whether a lineage of particles stayed in $\bar{A}_{0}$, exited $\bar{A}_{0}$ at 
some point in $k \in\{0, \ldots, n\}$ in $A$ or some other $\bar{A}_{j}, j \neq 0$ :

$$
\begin{aligned}
\mathbb{E}_{a}\left(\nu_{n}\left(e^{u \cdot}\right)\right) & =\mathbb{E}_{a}\left[\sum_{|x|=n} e^{u(\bar{V}(x)\rfloor)} \mathbb{1}_{T_{\bar{A}_{0}}(x)>n}\right] \\
& +\sum_{k=0}^{n} \mathbb{E}_{a}\left[\sum_{|x|=k} e^{u(V(x)-2\lfloor V(x) / 2\rfloor)} \mathbb{1}_{\left\{T_{\bar{A}_{0}}(x)>k-1\right\} \cap\{V(x) \in A\}}\right] \\
& +\sum_{k=0}^{n} \mathbb{E}_{a}\left[\sum_{|x|=n} e^{u(V(x)-2\lfloor V(x) / 2\rfloor)} \mathbb{1}_{\left\{T_{\bar{A}_{0}}(x)>k-1\right\} \cap\left\{V\left(x_{k}\right) \in \bar{A} \backslash \bar{A}_{0}\right\}}\right] \\
:= & \psi_{n}^{(1)}(a)+\psi_{n}^{(2)}(a)+\psi_{n}^{(3)}(a) .
\end{aligned}
$$

We wish to obtain the generating function of $\psi_{n}^{(2)}(a)$. As in development leading to Expression (57) one obtains

$$
\begin{aligned}
\sum_{n=0}^{\infty} t^{n} \psi_{n}^{(2)}(a):= & \hat{\psi}_{a}(t, A)=\int_{\cup_{j \leq-1} A_{j}} \mu e^{\mu z} d z \cdot \phi_{-}(t m) \\
& +e^{u} \int_{\cup_{j \geq 0} A_{j}} \mu e^{-\mu z} d z \cdot \phi_{+}(t m) \\
= & \sum_{j=-\infty}^{-1} \int_{2 j+1}^{2 j+2} \mu e^{\mu z} d z . \phi_{-}(t m)+e^{u} \sum_{j=0}^{\infty} \int_{2 j+1}^{2 j+2} \mu e^{-\mu z} d z . \phi_{+}(t m),
\end{aligned}
$$

leading to Expression (70).

We turn to generating function of $\psi_{n}^{(1)}(a)$. Since within $\bar{A}_{0}$ the branching process behaves as a classical branching random walk, and as $\lfloor V(x) / 2\rfloor=0$ while $V(x) \in \bar{A}_{0}$, one has

$$
\psi_{n}^{(1)}(a)=\mathbb{E}_{a}\left[\sum_{|x|=n} e^{u V(x)} \mathbb{1}_{T_{\bar{A}_{0}}(x)>n}\right] .
$$

Using Lemma 2, we have thus

$$
\begin{aligned}
\psi_{n}^{(1)}(a) & =m^{n} \mathbb{E}_{a}\left[e^{u S_{n}} \mathbb{1}_{\left\{S_{j} \in \bar{A}_{0}, j=0, \ldots, n\right\}}\right] \\
& =m^{n} \mathbb{E}_{a}\left[e^{u S_{n}}\right]-m^{n} \sum_{k=0}^{n} \mathbb{E}_{a}\left[e^{u S_{n}} \mathbb{1}_{\left\{S_{j} \in \bar{A}_{0}, j=0, \ldots, k-1, S_{k} \notin \bar{A}_{0}\right\}}\right] .
\end{aligned}
$$


Let us introduce $\mathbb{E}_{a}\left[e^{u S_{n}}\right]:=c_{n}(a)$, which, after straightforward computation, has the following expression:

$$
c_{n}(a)=e^{u a}\left(\frac{p}{m}+\frac{p \mathbb{E}(N)}{m} \frac{\mu^{2}}{\mu^{2}-u^{2}}\right)^{n}
$$

for $|u|<\mu$. Now using memoryless property of the exponential distribution:

$$
\begin{aligned}
& \mathbb{E}_{a}\left[e^{u S_{n}} \mathbb{1}_{\left\{S_{j} \in \bar{A}_{0}, j=0, \ldots, k-1, S_{k} \notin \bar{A}_{0}\right\}}\right] \\
= & \mathbb{E}\left[e^{u\left(S_{n-k}+W+1\right)}\right] \cdot \mathbb{P}_{a}\left[S_{j} \in \bar{A}_{0}, j=0, \ldots, k-1, S_{k}>1\right] \\
+ & \mathbb{E}\left[e^{u\left(S_{n-k}-W\right)}\right] \mathbb{P}_{a}\left[S_{j} \in \bar{A}_{0}, j=0, \ldots, k-1, S_{k}<0\right] \\
= & \int_{0}^{\infty} c_{n-k}(v+1) d \mathbb{P}_{W}(v) \cdot \mathbb{P}_{a}\left[T_{+}=k, T_{+}<T_{-}\right] \\
+ & \int_{0}^{\infty} c_{n-k}(-v) d \mathbb{P}_{W}(v) \cdot \mathbb{P}_{a}\left[T_{-}=k, T_{-}<T_{+}\right]
\end{aligned}
$$

where $W$ is distributed as $\mathcal{E}(\mu)$. Here we let $T_{+}$and $T_{-}$the respective right and left exit times of random walk $\left(S_{n}\right)_{n \in \mathbb{N}}$ out of $\bar{A}_{0}=[0,1)$. (72) then reads

$$
\begin{array}{r}
\psi_{n}^{(1)}(a)=m^{n} c_{n}(a)-m^{n} \sum_{k=0}^{n} \int_{0}^{\infty} c_{n-k}(v+1) d \mathbb{P}_{W}(v) \cdot \mathbb{P}_{a}\left[T_{+}=n, T_{+}<T_{-}\right] \\
-m^{n} \sum_{k=0}^{n} \int_{0}^{\infty} c_{n-k}(-v) d \mathbb{P}_{W}(v) \cdot \mathbb{P}_{a}\left[T_{-}=n, T_{-}<T_{+}\right]
\end{array}
$$

We deduce from the above that the generating function of $\psi_{n}^{(1)}(a)$ is

$$
\begin{aligned}
\sum_{n=0}^{\infty} t^{n} \psi_{n}^{(1)}(a):=\hat{\psi}_{a}\left(t, \bar{A}_{0}\right)=\hat{c}(m t, a) & -\int_{0}^{\infty} \hat{c}(m t, v+1) d \mathbb{P}_{W}(v) . \phi_{+}(m t) \\
& -\int_{0}^{\infty} \hat{c}(m t,-v) d \mathbb{P}_{W}(v) . \phi_{-}(m t)
\end{aligned}
$$

where $\hat{c}(s, a)$ is the following generating function

$$
\begin{array}{r}
\hat{c}(s, a):=\sum_{n=0}^{\infty} s^{n} c_{n}(a)=e^{u a} \frac{m\left(\mu^{2}-u^{2}\right)}{m\left(\mu^{2}-u^{2}\right)-s\left(p\left(\mu^{2}-u^{2}\right)+p \mathbb{E}(N) \mu^{2}\right)}, \\
|u|<\mu, \quad|s|<\frac{p}{m}+\frac{p \mathbb{E}(N)}{m} \frac{\mu^{2}}{\mu^{2}-u^{2}}
\end{array}
$$


which, plugged in (75), yields (69).

Finally, we turn to $\psi_{n}^{(3)}(a)$. Similarly to $(72)$ we write, using the Many to one Lemma (Lemma 2):

$$
\begin{aligned}
& \mathbb{E}_{a}\left[\sum_{|x|=n} e^{u(V(x)-2\lfloor V(x) / 2\rfloor)} \mathbb{1}_{\left\{T_{\bar{A}_{0}}>k-1\right\} \cap\left\{V\left(x_{k}\right) \in \bar{A} \backslash \bar{A}_{0}\right\}}\right] \\
= & \mathbb{E}_{a}\left[\sum_{|x|=n} e^{u(V(x)-2\lfloor V(x) / 2\rfloor)} \mathbb{1}_{\left\{T_{\bar{A}_{0}}>k-1\right\} \cap\left\{V\left(x_{j}\right) \in \bar{A} \backslash \bar{A}_{0}, j=k, \ldots, n\right\}}\right] \\
+ & \sum_{p=k}^{n} \mathbb{E}_{a}\left[\sum_{|x|=n} e^{u\left(V\left(x_{p}\right)-2\left\lfloor V\left(x_{p}\right) / 2\right\rfloor\right.} \mathbb{1}_{\left\{T_{\bar{A}_{0}}>k-1\right\} \cap\left\{V\left(x_{j}\right) \in \bar{A} \backslash \bar{A}_{0}, j=k, \ldots, p-1, V\left(x_{p}\right) \in A\right\}}\right] \\
= & m^{n} \mathbb{E}_{a}\left[e^{u\left(S_{n}-2\left\lfloor S_{n} / 2\right\rfloor\right)} \mathbb{1}_{\left\{S_{j} \in \bar{A}_{0}, j=0, \ldots, k-1\right\} \cap\left\{S_{j} \in \bar{A} \backslash \bar{A}_{0}, j=k, \ldots, n\right\}}\right] \\
+ & m^{n} \sum_{p=k}^{n} \mathbb{E}_{a}\left[e^{u\left(S_{p}-2\left\lfloor S_{p} / 2\right\rfloor\right)} \mathbb{1}_{\left\{S_{j} \in \bar{A}_{0}, j=0, \ldots, k-1\right\} \cap\left\{S_{j} \in \bar{A} \backslash \bar{A}_{0}, j=k, \ldots, p-1, S_{p} \in A\right\}}\right] . \quad \text { (76) }
\end{aligned}
$$

Now in the second term in the above equality one has, considering both cases according to whether $S_{k} \in \bar{A} \backslash \bar{A}_{0}$ is positive or negative:

$$
\begin{aligned}
& \mathbb{E}_{a}\left[e^{u\left(S_{p}-2\left\lfloor S_{p} / 2\right\rfloor\right)} \mathbb{1}_{\left\{S_{j} \in \bar{A}_{0}, j=0, \ldots, k-1\right\} \cap\left\{S_{j} \in \bar{A} \backslash \bar{A}_{0}, j=k, \ldots, p-1, S_{p} \in A\right\}}\right] \\
= & \mathbb{E}_{a}\left[e^{u\left(S_{p}-2\left\lfloor S_{p} / 2\right\rfloor\right)} \mathbb{1}_{\left\{S_{j} \in \bar{A}_{0}, j=0, \ldots, k-1\right\} \cap\left\{S_{k} \in \bigcup_{r \geq 1} \bar{A}_{r}, S_{j} \in \bar{A} \backslash \bar{A}_{0}, j=k+1, \ldots, p-1, S_{p} \in A\right\}}\right] \\
+ & \mathbb{E}_{a}\left[e^{u\left(S_{p}-2\left\lfloor S_{p} / 2\right\rfloor\right)} \mathbb{1}_{\left\{S_{j} \in \bar{A}_{0}, j=0, \ldots, k-1\right\} \cap\left\{S_{k} \in \bigcup_{r \leq-1} \bar{A}_{r}, S_{j} \in \bar{A} \backslash \bar{A}_{0}, j=k, \ldots, p-1, S_{p} \in A\right\}}\right] .(77
\end{aligned}
$$

The following is obtained according to the Markov property, the memoryless property of exponential distribution, then again Lemma 2:

$$
\begin{aligned}
& m^{n} \mathbb{E}_{a}\left[e^{u\left(S_{p}-2\left\lfloor S_{p} / 2\right\rfloor\right)} \mathbb{1}_{\left\{S_{j} \in \bar{A}_{0}, j=0, \ldots, k-1\right\} \cap\left\{S_{k} \in \bigcup_{r \geq 1} \bar{A}_{r}, S_{j} \in \bar{A} \backslash \bar{A}_{0}, j=k+1, \ldots, p-1, S_{p} \in A\right\}}\right] \\
= & m^{n} \mathbb{E}\left[e^{u\left(\left(S_{p-k}+W\right)-2\left\lfloor\left(S_{p-k}+W\right) / 2\right\rfloor\right)} \mathbb{1}_{\left\{W \in \bigcup_{r \geq 1} \bar{A}_{r}, W+S_{j} \in \bar{A} \backslash \bar{A}_{0}, j=1, \ldots, p-k-1, W+S_{p-k} \in A\right\}}\right] \\
& . \mathbb{P}_{a}\left[T_{+}=k, T_{+}<T_{-}\right] \\
= & m^{k} \int_{\bigcup_{r \geq 1} \bar{A}_{r}} \mathbb{E}_{v}\left[\sum_{|x|=n-k} e^{u\left(V\left(x_{p-k}\right)-2\left\lfloor V\left(x_{p-k}\right) / 2\right\rfloor\right)} \mathbb{1}_{\left\{V\left(x_{j}\right) \in \bar{A} \backslash \bar{A}_{0}, j=1, \ldots, p-k-1, V\left(x_{p-k}\right) \in A\right\}}\right] \\
& d \mathbb{P}_{W}(v) . \mathbb{P}_{a}\left[T_{+}=k, T_{+}<T_{-}\right]
\end{aligned}
$$


where $W=1+Z$ where $Z$ is a generic $\mathcal{E}(\mu)$ distributed r.v. Treating similarly second term in the right-hand side of (77) yields a similar formula as (78) and entails the following

$$
\begin{aligned}
& \mathbb{E}_{a}\left[e^{u\left(S_{p}-2\left\lfloor S_{p} / 2\right\rfloor\right)} \mathbb{1}_{\left\{S_{j} \in \bar{A}_{0}, j=0, \ldots, k-1\right\} \cap\left\{S_{j} \in \bar{A} \backslash \bar{A}_{0}, j=k, \ldots, p-1, S_{p} \in A\right\}}\right] \\
= & m^{k} \int_{\bigcup_{r \geq 1} \bar{A}_{r}} \mathbb{E}_{v}\left[\sum_{|x|=n-k} e^{u\left(V\left(x_{p-k}\right)-2\left\lfloor V\left(x_{p-k}\right) / 2\right\rfloor\right)} \mathbb{1}_{\left\{V\left(x_{j}\right) \in \bar{A} \backslash \bar{A}_{0}, j=1, \ldots, p-k-1, V\left(x_{p-k}\right) \in A\right\}}\right] \\
& d \mathbb{P}_{W}(v) \cdot \mathbb{P}_{a}\left[T_{+}=k, T_{+}<T_{-}\right] \\
+ & m^{k} \int_{\bigcup_{r \leq-1} \bar{A}_{r}} \mathbb{E}_{v}\left[\sum_{|x|=n-k} e^{u\left(V\left(x_{p-k}\right)-2\left\lfloor V\left(x_{p-k}\right) / 2\right\rfloor\right)} \mathbb{1}_{\left\{V\left(x_{j}\right) \in \bar{A} \backslash \bar{A}_{0}, j=1, \ldots, p-k-1, V\left(x_{p-k}\right) \in A\right\}}\right] \\
& d \mathbb{P}_{W^{\prime}}(v) \cdot \mathbb{P}_{a}\left[T_{-}=k, T_{-}<T_{+}\right]
\end{aligned}
$$

where $W^{\prime}$ is such that $-W^{\prime}$ is $\mathcal{E}(\mu)$ distributed. Finally, one has similarly that the first term on the right-hand side of (76) verifies

$$
\begin{aligned}
& m^{n} \mathbb{E}_{a}\left[e^{u\left(S_{n}-2\left\lfloor S_{n} / 2\right\rfloor\right)} \mathbb{1}_{\left\{S_{j} \in \bar{A}_{0}, j=0, \ldots, k-1\right\} \cap\left\{S_{j} \in \bar{A} \backslash \bar{A}_{0}, j=k, \ldots, n\right\}}\right] \\
= & m^{k} \int_{\bigcup_{r \geq 1} \bar{A}_{r}} \mathbb{E}_{v}\left[\sum_{|x|=n-k} e^{u(V(x)-2\lfloor V(x) / 2\rfloor)} \mathbb{1}_{\left\{V\left(x_{j}\right) \in \bar{A} \backslash \bar{A}_{0}, j=1, \ldots, n-k\right\}}\right] d \mathbb{P}_{W}(v) \\
& . \mathbb{P}_{a}\left[T_{+}=k, T_{+}<T_{-}\right] \\
+ & m^{k} \int_{\bigcup_{r \leq-1} \bar{A}_{r}} \mathbb{E}_{v}\left[\sum_{|x|=n-k} e^{u(V(x)-2\lfloor V(x) / 2\rfloor)} \mathbb{1}_{\left\{V\left(x_{j}\right) \in \bar{A} \backslash \bar{A}_{0}, j=1, \ldots, n-k\right\}}\right] d \mathbb{P}_{W^{\prime}}(v) \\
& . \mathbb{P}_{a}\left[T_{-}=k, T_{-}<T_{+}\right] .
\end{aligned}
$$

Gathering (79) and (80) and plugging into (76) yields

$$
\begin{aligned}
\psi_{n}^{(3)}(a) & =\sum_{k=0}^{n} m^{k} \int_{\bigcup_{r \geq 1} \bar{A}_{r}} \mathbb{E}_{v}\left(\nu_{n-k}\left(e^{u \cdot}\right)\right) d \mathbb{P}_{W}(v) \cdot \mathbb{P}_{a}\left[T_{+}=k, T_{+}<T_{-}\right] \\
& +\sum_{k=0}^{n} m^{k} \int_{\bigcup_{r \leq-1} \bar{A}_{r}} \mathbb{E}_{v}\left(\nu_{n-k}\left(e^{u \cdot}\right)\right) d \mathbb{P}_{W^{\prime}}(v) . \mathbb{P}_{a}\left[T_{-}=k, T_{-}<T_{+}\right] .
\end{aligned}
$$

Finally, noticing that $\int_{\bigcup_{r \leq-1} \bar{A}_{r}} \mathbb{E}_{v}\left(\nu_{n-k}\left(e^{u \cdot}\right)\right) d \mathbb{P}_{W^{\prime}}(v)=\int_{\bigcup_{r>1} \bar{A}_{r}} \mathbb{E}_{2-v}\left(\nu_{n-k}\left(e^{u \cdot}\right)\right) d \mathbb{P}_{W}(v)$, decomposition (71) alongside Expressions (69), (70), as well as decomposition 
(80), yield the following integral equations for generating function $\hat{\psi}(t,$.$) :$

$$
\begin{aligned}
\hat{\psi}(t, a) & =\hat{\psi}_{a}\left(t, \bar{A}_{0}\right)+\hat{\psi}_{a}(t, A) \\
& +\int_{\bigcup_{r \geq 1} \bar{A}_{r}} \hat{\psi}(t, v) d \mathbb{P}_{1+W}(v) \cdot \phi_{+}(m t)+\int_{\bigcup_{r \leq-1} \bar{A}_{r}} \hat{\psi}(t, v) d \mathbb{P}_{-W}(v) \phi_{-}(m t)
\end{aligned}
$$

which, by straightforward computation, yields (68).

Uniqueness of solution to (68). We use here the notation $\phi_{+, a}$ (resp. $\left.\phi_{-a}\right)$ for $\phi_{+}\left(\operatorname{resp} \phi_{-}\right)$to make explicit the dependence in $a$. Let us introduce the following operator $\mathcal{A}$ defined on the set of continuous functions from $\bar{A}_{0}$ to $\mathbb{R}$ by

$$
\mathcal{A} f(a):=\int_{\bar{A}_{0}} f(v) \mu e^{-\mu v} d v \frac{e^{-\mu}}{1-e^{-2 \mu}} \phi_{+, a}(m t)+\int_{\bar{A}_{0}} f(v) \mu e^{\mu v} d v \frac{e^{-2 \mu}}{1-e^{-2 \mu}} \phi_{-, a}(m t),
$$

$a \in \bar{A}_{0}$, (where $t$ is fixed), equipped with norm $\|f\|:=\sup _{a \in \bar{A}_{0}}|f(a)|$. A short computation shows that $\mathcal{A}$ satisfies the following inequality

$$
\|\mathcal{A} f\| \leq C .\|f\|
$$

where

$$
\begin{aligned}
C & :=\sup _{a \in \bar{A}_{0}}\left|\left(1-e^{-\mu}\right) \frac{e^{-\mu}}{1-e^{-2 \mu}} \phi_{+, a}(m t)+\left(e^{\mu}-1\right) \frac{e^{-2 \mu}}{1-e^{-2 \mu}} \phi_{-, a}(m t)\right| \\
& \leq\left[\left(1-e^{-\mu}\right) \frac{e^{-\mu}}{1-e^{-2 \mu}}+\left(e^{\mu}-1\right) \frac{e^{-2 \mu}}{1-e^{-2 \mu}}\right] \max \left(\left\|\phi_{+, .}(m t)\right\|,\left\|\phi_{-, .}(m t)\right\|\right) \\
& =\frac{2 e^{-\mu}}{1+e^{-\mu}} \max \left(\left\|\phi_{+, .}(m t)\right\|,\left\|\phi_{-, .}(m t)\right\|\right),
\end{aligned}
$$

a quantity which is strictly less than 1 since $\frac{2 e^{-\mu}}{1+e^{-\mu}},\left\|\phi_{+, .}(m t)\right\|$ and $\left\|\phi_{-, .}(m t)\right\|$ are all quantities upper bounded by 1 . Thus $\mathcal{A}$ is a contracting operator, and Equation (68), which can also be written

$$
\hat{\psi}(t, a)=\hat{\psi}_{a}\left(t, \bar{A}_{0}\right)+\hat{\psi}_{a}(t, A)+(\mathcal{A} \hat{\psi}(t, .))(a), \quad a \in \bar{A}_{0},
$$

admits a unique solution.

Acknowledgment. The authors would like to thank the anonymous referee for his/her remarks leading to an improved version of the paper. This work was partially supported by Projet Région "Modélisation Mathématique des Éléments Transposables". 
[1] Aïdékon, E. (2013). Convergence in law of the minimum of a branching random walk. Ann. Probab., 41(3A):1362-1426.

[2] K. B. Athreya and P. E. Ney (2004). Branching processes. Dover Publications, Inc., Mineola, NY. Reprint of the 1972 original [Springer, New York; MR0373040].

[3] Horn, R. A. and Johnson, C. R. (2013). Matrix analysis. Cambridge University Press, Cambridge, second edition.

[4] Jacobsen, M. (2011). Exit times for a class of random walks: exact distribution results. J. Appl. Probab., 48A(New frontiers in applied probability: a Festschrift for Soren Asmussen):51-63.

[5] Kingman, J.F.C. (1975). The first birth problem for an age dependent branching process. Ann. Probab., 3:790-801.

[6] Kahane, J.-P. and Peyrière, J. (1976). Sur certaines martingales de Benoit Mandelbrot. Advances in Math., 22(2):131-145.

[7] Kurtz, T., Lyons, R., Pemantle, R., and Peres, Y. (1997). A conceptual proof of the Kesten-Stigum theorem for multi-type branching processes. In Classical and modern branching processes (Minneapolis, MN, 1994), volume 84 of IMA Vol. Math. Appl., pages 181-185. Springer, New York.

[8] Mallein, B. (2015). Maximal displacement in a branching random walk through interfaces. Electron. J. Probab, 20(68):1-40.

[9] Shi, Z. (2012). Branching Random Walks, École d'Été de Probabilités de Saint-Flour, XLII. Springer International Publishing, Series Volume 2151. 